\title{
Small- $x$ phenomenology at the LHC and beyond: HELL 3.0 and the case of the Higgs cross section
}

\author{
Marco Bonvini ${ }^{\mathrm{a}}$ iD \\ INFN, Sezione di Roma 1, Piazzale Aldo Moro 5, 00185 Rome, Italy
}

Received: 23 July 2018 / Accepted: 6 October 2018 / Published online: 17 October 2018

(C) The Author(s) 2018

\begin{abstract}
Small- $x$ resummation has been proven recently to be a crucial ingredient for describing small- $x$ HERA data, and the inclusion of small- $x$ resummation in parton distribution function (PDF) determination has a sizeable effect on the PDFs even at the electroweak scale. In this work we explore the implications of small- $x$ resummation at the large hadron collider (LHC) and at a future circular collider (FCC). We construct the theoretical machinery for resumming physical inclusive observables at hadron colliders, and describe its implementation in the public code HELL 3.0. We focus on Higgs production in gluon fusion as a prototypical example, both because it is sensitive to small- $x$ gluons and because of its importance for the LHC physics programme. We find that adding small- $x$ resummation to the $\mathrm{N}^{3} \mathrm{LO}$ Higgs production cross section can lead to an increase of up to $10 \%$ at FCC, while the effect is smaller $(+1 \%)$ at LHC but still important to achieve a high level of precision.
\end{abstract}

\section{Contents}

1 Introduction . . . . . . . . . . . . . 1

2 Hadron-hadron collider processes at high-energy . . 2

2.1 Resummation formalism with two incoming gluon legs ............... . . 2

2.2 The evolution function . . . . . . . . . . . 4

2.3 Equivalence to the impact factor formulation . . 6

2.4 Expansion and matching to fixed order . . . . . 6

2.5 The first few orders of the anomalous dimension at $\mathrm{LL}^{\prime}$ and NLL . . . . . . . . . . . . . . . 8

3 Resummed Higgs cross section at the LHC and beyond

3.1 Construction of the fixed-order result at small $x$ with top mass dependence . . . . . . . . . . .

3.2 Impact of high-energy resummation at parton level ................. 15

a e-mail: marco.bonvini@ roma1.infn.it
3.3 Impact of high-energy resummation on the cross section ................. 18

4 Conclusions .................. 25

Appendix A: Off-shell coefficient function for Higgs production ............... 26

A.1: Off-shell coefficient function in suitable variables ................ 26 26

A.2: Impact factor and its expansion coefficients . . 28 References ................. 29

\section{Introduction}

With the discovery of the Higgs boson, the Standard Model (SM) has been established as a successful theory of particle physics. While the SM cannot be the definitive theory, direct evidence of physics beyond the SM has not (yet) been observed at the LHC. The search for new phenomena beyond the SM at hadron colliders may be pursued by testing the SM to high precision, which is becoming possible thanks to the huge amount and excellent quality of the data collected by the LHC. To keep up, theoretical predictions must reach and possibly surpass the precision of the measurements. On the one hand, this requires refined theoretical predictions for the partonic cross sections for the processes of interest, which may be obtained by higher order computations, e.g. next-tonext-to-leading order (NNLO) or even next-to-next-to-nextto-leading order $\left(\mathrm{N}^{3} \mathrm{LO}\right)$ in some cases, and by the all-order resummation of important classes of logarithmic contributions. On the other hand, accurate and precise theoretical predictions for LHC processes require high-quality parton distribution functions (PDFs).

Recently, an important step forward towards improved determination of PDFs has been achieved in Refs. [1,2], where the resummation of small- $x$ (high-energy) logarithms at next-to-leading logarithmic (NLL) accuracy as implemented in the public code HELL [3,4] has been included in PDF evolution and in the theoretical predictions of DIS observables. Small- $x$ resummation has the important role of 
stabilizing the behaviour of DGLAP splitting functions at small $x$, which otherwise is compromised by powers of $\log \frac{1}{x}$. In particular, the first manifest instability appears at NNLO, and thus PDFs determined with NNLO theory are rather different to those determined with NNLO theory improved by NLL small- $x$ resummation. This difference, determined at low $Q^{2}$ where the small- $x$ HERA data lie, persists and is actually enlarged by DGLAP evolution at larger scales. As a result, resummed PDFs at the electroweak scale are very different from the NNLO ones at small $x$.

This raises an important question: how does this large effect impact LHC precision phenomenology? To properly answer, we need to compare fixed-order prediction with fixed-order (NNLO) PDFs to resummed predictions with resummed (NNLO + NLL) PDFs. While NNLO + NLL PDFs are now available, resummed predictions for LHC observables did not exist, or at least not in a format which makes them immediately usable for phenomenology. It is the goal of this paper to provide the theoretical setup to perform this resummation for inclusive observables with the public code HELL. The resummation of differential observables with HELL is left to future work.

As a first example of application of this setup, we will consider Higgs production in gluon fusion. Being initiated by two initial-state gluons, this process is very sensitive to the gluon PDF. Moreover, it is known that the inclusive Higgs cross section is dominated by contributions close to partonic threshold, which in turn implies that the gluon PDF contributes mostly at small $x$. In addition, the inclusive Higgs cross section in gluon fusion is known to $\mathrm{N}^{3} \mathrm{LO}$ [5-8], so we will provide all the ingredients to properly match small- $x$ resummation of a physical process to $\mathrm{N}^{3} \mathrm{LO}$ for the first time. We then investigate the phenomenological implications of small- $x$ resummation in Higgs production at the LHC, and to enlarge the sensitivity to the PDFs at small $x$ also at higherenergy colliders, namely high-energy LHC (HE-LHC) and a future circular hadron-hadron collider (FCC-hh).

The structure of the paper is the following. In Sect. 2 we derive the formalism for small- $x$ resummation of inclusive cross sections with two hadrons in the initial state. We discuss its implementation in the HELL code, and compare it to the original formulation [9] in the Altarelli-Ball-Forte (ABF) formalism [10-15]. We provide all the ingredients for matching small- $x$ resummation in the partonic coefficient functions to $\mathrm{N}^{3} \mathrm{LO}$. In Sect. 3 we move to Higgs production, and present first how the fixed-order cross section can be constructed to treat correctly the small- $x$ behaviour at NNLO and $\mathrm{N}^{3} \mathrm{LO}$, and then the effect of adding small- $x$ resummation both at parton level and at the level of the physical cross section. We then draw our conclusions in Sect. 4, and collect technical details in Appendix A. This work represents a follow up of Refs. $[1,3,4,16]$, and provides the foundations of Ref. [17].

\section{Hadron-hadron collider processes at high-energy}

The resummation of small- $x$ logarithms in physical processes requires both using PDFs which include small- $x$ resummation in their determination and evolution, and resumming to all orders the $\log \frac{1}{x}$ contributions in the partonic coefficient functions. The latter resummation, which is the subject of this section, is based on the so-called $k_{\mathrm{t}}$ factorization theorem, where the non-perturbative proton dynamics is factorized in parton distribution functions which depend on both the longitudinal momentum fraction $x$ of the parton and its transverse momentum $k_{\mathrm{t}}$ [18-23]. Relating this $k_{\mathrm{t}}$-dependent PDFs to the usual collinear PDFs it is possible to resum the leading non-vanishing tower of small- $x$ logarithms to all orders in the collinearly factorized partonic coefficient functions.

Another important ingredient for a stable small- $x$ resummation is the inclusion to all orders of a class of subleading contributions originating from the running of the strong coupling $\alpha_{s}$ [9, 15]. In Ref. [3] the approach of Refs. [9, 15] has been rederived and reformulated in a simpler and more general way, and proven to be identical to the original formulation under specific assumptions. The new formulation of Ref. [3] has been implemented in the computer code HELL [3,4], and it is very convenient from the analytical and numerical points of view, making the resummation of new processes and their inclusion in HELL rather straightforward. In Ref. [3], and subsequently in Ref. [4], this new formalism has been presented and used only for processes with a single hadron in the initial state, and specifically the deep inelastic scattering (DIS) process. In this section we extend the formulation to processes with two hadrons in the initial state, relevant for hadron-hadron colliders such as the LHC. This extension was already presented in the original formulation in Refs. [9, 24]; in this section we will also show that our formulation, which is more general, reduces to the original one under the same assumptions considered for the single-hadron case.

\subsection{Resummation formalism with two incoming gluon legs}

We consider a hadron-collider process which is gluon-gluon initiated. The typical and cleanest example, which we will consider in greater detail later in Sect. 3, is Higgs production in gluon fusion, whose leading order diagram is depicted in Fig. 1. Other examples for which the results of this section will be relevant are, e.g., top-pair production and jet production.

We will assume that there are no collinear singularities to be subtracted at resummed level. Namely, the lowest order diagram with two gluons in the initial state must be finite without any collinear subtraction. Indeed, in order for a collinear singularity to be present, at least one of the gluons must split into a pair of quarks, one of which participates to the hard interaction. In other words, it must be possible to cut 


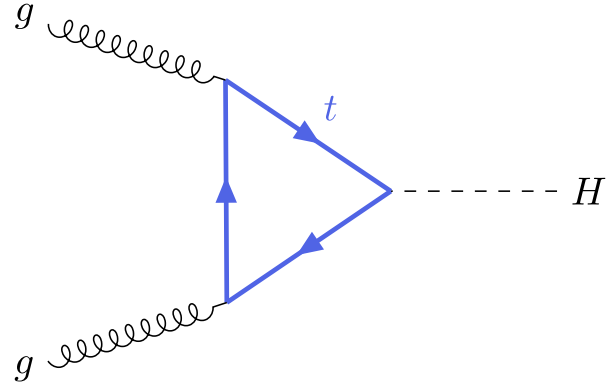

Fig. 1 Leading order diagram for Higgs production in gluon fusion at hadron-hadron colliders. The quark running in the loop is predominantly a top

a quark line such that the diagram factorizes into a gluon splitting to quarks and a gluon-quark initiated subgraph. Therefore, in presence of collinear singularities in a $g g$ initiated diagram, there must exist a lower order diagram which is $g q$ initiated. But if this is the case, the resummation of the $g g$ initiated process is subleading logarithmic with respect to the resummation of the $g q$ initiated process, due to the extra power of $\alpha_{s}$ and no logarithm in the $g \rightarrow q \bar{q}$ splitting. Thus, at the leading non-vanishing logarithmic accuracy, contribution with two initial state gluons which require collinear subtractions do not contribute. This is the case for instance of Drell-Yan production, where indeed at lowest logarithmic order only the $g q$ (and $q q$ ) channels contribute [24]. Of course, it is well possible that such $g q$ channel contains itself a collinear singularity (as it happens in the Drell-Yan case). However, this process has a single gluon in the initial state, and the treatment is identical to the DIS case already discussed in Ref. [3].

Let us then focus on the cross section $\sigma$ of a gluon-gluon initiated (at lowest order) process without collinear singularities, such as Higgs production, in hadron-hadron collision. In order to simplify the treatment, we take the Mellin transform of the cross section as

$\sigma\left(N, Q^{2}\right)=\int_{0}^{1} d \tau \tau^{N} \sigma\left(\tau, Q^{2}\right)$

where $\tau=Q^{2} / s$, with $Q$ the hard scale of the process (e.g., the Higgs mass) and $\sqrt{s}$ the collider center-of-mass energy. The cross section in collinear factorization can be written in Mellin $N$ space as the sum over partonic channels of simple products,

$$
\begin{aligned}
\sigma\left(N, Q^{2}\right)= & \sigma_{0}\left(N, Q^{2}\right) \sum_{i, j=g, q} C_{i j}\left(N, \alpha_{s}, \frac{\mu_{\mathrm{F}}^{2}}{Q^{2}}\right) \\
& \times f_{i}\left(N, \mu_{\mathrm{F}}^{2}\right) f_{j}\left(N, \mu_{\mathrm{F}}^{2}\right),
\end{aligned}
$$

where $C_{i j}$ are the collinearly factorized coefficient functions and $f_{k}$ the collinear PDFs, which depend on the factorization scale $\mu_{\mathrm{F}} \sim Q$. The strong coupling $\alpha_{s}$ is in general evaluated at the renormalization scale $\mu_{\mathrm{R}}$, which implies that there are logarithms of $\mu_{\mathrm{R}} / Q$ in the coefficient function to compensate its dependence; however, at the leading logarithmic accuracy we will consider, the $\mu_{\mathrm{R}}$ dependence is subleading, and we therefore omit it to simplify the notation. The factor $\sigma_{0}$ is chosen such that the coefficients functions are dimensionless, and normalized to 1 at LO in the dominant channel (supposed to be the $g g$ channel in our case). In the high-energy limit, there is no need to distinguish the individual quarks, as they always contribute in the singlet combination. Thus, in this section, we will assume that the index $q$ refers to the whole singlet PDF. ${ }^{1}$

In the high-energy limit, the cross section can be also written according to the $k_{\mathrm{t}}$ factorization theorem, which gives

$$
\begin{aligned}
\sigma\left(N, Q^{2}\right)= & \sigma_{0}\left(N, Q^{2}\right) \int d k_{\mathrm{t} 1}^{2} d k_{\mathrm{t} 2}^{2} \mathcal{C}\left(N, \frac{k_{\mathrm{t} 1}^{2}}{Q^{2}}, \frac{k_{\mathrm{t} 2}^{2}}{Q^{2}}, \alpha_{s}\right) \\
& \times \mathcal{F}_{g}\left(N, k_{\mathrm{t} 1}^{2}\right) \mathcal{F}_{g}\left(N, k_{\mathrm{t} 2}^{2}\right) .
\end{aligned}
$$

Here, $\mathcal{F}_{g}$ is the $k_{\mathrm{t}}$-dependent gluon $\mathrm{PDF}$, and $\mathcal{C}$ is the partonic coefficient function computed with two off-shell incoming gluons, the off-shellness being $k_{\mathrm{t}}^{2}$. Obviously, the off-shell coefficient function is symmetric for the exchange of the two virtualities, $k_{\mathrm{t} 1}^{2} \leftrightarrow k_{\mathrm{t} 2}^{2}$. The $k_{\mathrm{t}}$-dependent gluon PDF can be related to the collinear PDFs through the relation [3,23,24]

$$
\begin{aligned}
\mathcal{F}_{g}\left(N, k_{\mathrm{t}}^{2}\right)= & \mathcal{U}\left(N, k_{\mathrm{t}}^{2}, \mu_{\mathrm{F}}^{2}\right) f_{g}\left(N, \mu_{\mathrm{F}}^{2}\right) \\
& +\frac{C_{F}}{C_{A}}\left[\mathcal{U}\left(N, k_{\mathrm{t}}^{2}, \mu_{\mathrm{F}}^{2}\right)-\delta\left(k_{\mathrm{t}}^{2}\right)\right] f_{q}\left(N, \mu_{\mathrm{F}}^{2}\right),
\end{aligned}
$$

where $\mathcal{U}$ is a function which is factorization scheme dependent. $^{2}$ In the $Q_{0} \overline{\mathrm{MS}}$ scheme $[21,23,25,26]$ usually considered in the high-energy regime, and adopted also here, it is given by

$\mathcal{U}_{Q_{0} \overline{\mathrm{MS}}}\left(N, k_{\mathrm{t}}^{2}, \mu_{\mathrm{F}}^{2}\right)=\frac{d}{d k_{\mathrm{t}}^{2}} U\left(N, k_{\mathrm{t}}^{2}, \mu_{\mathrm{F}}^{2}\right)$,

with [3]

$U\left(N, k_{\mathrm{t}}^{2}, \mu_{\mathrm{F}}^{2}\right)=\exp \int_{\mu_{\mathrm{F}}^{2}}^{k_{\mathrm{t}}^{2}} \frac{d \mu^{2}}{\mu^{2}} \gamma_{+}\left(N, \alpha_{s}\left(\mu^{2}\right)\right)$,

\footnotetext{
1 With this assumption Eq. (2.2) is incomplete as it misses non-singlet contributions; however, this is irrelevant for the present discussion, which is focussed on the high-energy limit.

${ }^{2}$ We observe that in Ref. [3] the same equation was written in terms of the "plus" eigenvector PDF, see Eq. (3.3) there. However, that expression misses a contribution $+\delta\left(k_{\mathrm{t}}^{2}\right) f_{-}\left(N, Q^{2}\right)$ in terms of the "minus" eigenvector PDF, which produces the $\delta\left(k_{\mathrm{t}}^{2}\right)$ term in Eq. (2.4). The results of Ref. [3] are unaffected; the only effect of that deficiency is that $C_{-}$ appearing in Eq. (3.26) of that reference could be written in terms of the off-shell coefficient function. However, that contribution is purely NLO, and could thus be extracted from the fixed-order computation.
} 
where $\gamma_{+}$is the (small- $x$ resummed) eigenvalue of the anomalous dimension singlet matrix which is singular at small $x$. With respect to Ref. [3], we are slightly changing the notation for the evolution function $U$, to extend it to the case in which $\mu_{\mathrm{F}}$ is different from the hard scale $Q$. More details on the actual form of the evolution function $U$ and on the anomalous dimension used in its definition are given later in Sect. 2.2.

Plugging Eq. (2.4) into Eq. (2.3) and comparing with Eq. (2.2), we find a relation between the coefficient functions in collinear factorization and the off-shell coefficient functions,

$$
\begin{aligned}
C_{g g}\left(N, \alpha_{s}, \frac{\mu_{\mathrm{F}}^{2}}{Q^{2}}\right)= & \int d \xi_{1} d \xi_{2} \mathcal{C}\left(N, \xi_{1}, \xi_{2}, \alpha_{s}\right) \\
& \times \frac{d}{d \xi_{1}} U\left(N, Q^{2} \xi_{1}, \mu_{\mathrm{F}}^{2}\right) \\
& \times \frac{d}{d \xi_{2}} U\left(N, Q^{2} \xi_{2}, \mu_{\mathrm{F}}^{2}\right), \\
C_{q g}\left(N, \alpha_{s}, \frac{\mu_{\mathrm{F}}^{2}}{Q^{2}}\right)= & \frac{C_{F}}{C_{A}} \int d \xi_{1} d \xi_{2} \mathcal{C}\left(N, \xi_{1}, \xi_{2}, \alpha_{s}\right) \\
& \times \frac{d}{d \xi_{1}} U\left(N, Q^{2} \xi_{1}, \mu_{\mathrm{F}}^{2}\right) \\
& \times\left[\frac{d}{d \xi_{2}} U\left(N, Q^{2} \xi_{2}, \mu_{\mathrm{F}}^{2}\right)-\delta\left(\xi_{2}\right)\right], \\
C_{q q}\left(N, \alpha_{s}, \frac{\mu_{\mathrm{F}}^{2}}{Q^{2}}\right)= & \left(\frac{C_{F}}{C_{A}}\right)^{2} \int d \xi_{1} d \xi_{2} \mathcal{C}\left(N, \xi_{1}, \xi_{2}, \alpha_{s}\right) \\
& \times\left[\frac{d}{d \xi_{1}} U\left(N, Q^{2} \xi_{1}, \mu_{\mathrm{F}}^{2}\right)-\delta\left(\xi_{1}\right)\right] \\
& \times\left[\frac{d}{d \xi_{2}} U\left(N, Q^{2} \xi_{2}, \mu_{\mathrm{F}}^{2}\right)-\delta\left(\xi_{2}\right)\right],
\end{aligned}
$$

where we have introduced the dimensionless variables

$\xi=\frac{k_{\mathrm{t}}^{2}}{Q^{2}}$.

Introducing the "auxiliary" coefficient function

$$
\begin{aligned}
C_{\text {aux }}\left(N, \alpha_{s}, \frac{\mu_{\mathrm{F}}^{2}}{Q^{2}}\right)= & -\int d \xi_{1} d \xi_{2} \mathcal{C}\left(N, \xi_{1}, \xi_{2}, \alpha_{s}\right) \\
& \times \frac{d}{d \xi_{1}} U\left(N, Q^{2} \xi_{1}, \mu_{\mathrm{F}}^{2}\right) \delta\left(\xi_{2}\right) \\
= & -\int d \xi \mathcal{C}\left(N, \xi, 0, \alpha_{s}\right) \\
& \times \frac{d}{d \xi} U\left(N, Q^{2} \xi, \mu_{\mathrm{F}}^{2}\right),
\end{aligned}
$$

we can rewrite the quark coefficient functions as

$$
\begin{aligned}
C_{q g}\left(N, \alpha_{s}, \frac{\mu_{\mathrm{F}}^{2}}{Q^{2}}\right)= & \frac{C_{F}}{C_{A}}\left[C_{g g}\left(N, \alpha_{s}, \frac{\mu_{\mathrm{F}}^{2}}{Q^{2}}\right)\right. \\
& \left.+C_{\mathrm{aux}}\left(N, \alpha_{s}, \frac{\mu_{\mathrm{F}}^{2}}{Q^{2}}\right)\right], \\
C_{q q}\left(N, \alpha_{s}, \frac{\mu_{\mathrm{F}}^{2}}{Q^{2}}\right)= & \left(\frac{C_{F}}{C_{A}}\right)^{2}\left[C_{g g}\left(N, \alpha_{s}, \frac{\mu_{\mathrm{F}}^{2}}{Q^{2}}\right)\right. \\
& \left.+2 C_{\text {aux }}\left(N, \alpha_{s}, \frac{\mu_{\mathrm{F}}^{2}}{Q^{2}}\right)+\mathcal{C}\left(N, 0,0, \alpha_{s}\right)\right] .
\end{aligned}
$$

These expressions, already derived e.g. in Ref. [27], allow us to express both quark coefficient functions in terms of the gluon one and of the auxiliary function. Thus, from now on we will focus on the functions $C_{g g}$, Eq. (2.7a), and $C_{\text {aux }}$, Eq. (2.9).

\subsection{The evolution function}

The evolution function $U$, Eq. (2.6), is a key object for small$x$ resummation in partonic coefficient functions. Indeed Eqs. (2.7) encode small- $x$ resummation thanks to the form of $U$, which contains the leading small- $x$ logarithms to all orders, provided the anomalous dimension in there is itself accurate at least at LL. We thus now recall here some properties of this function already presented in Refs. [3,4], with particular focus on its $\mu_{\mathrm{F}}$ dependence that we are now including.

First, we observe that the anomalous dimension in Eq. (2.6) is integrated between $\mu_{\mathrm{F}}$ and $k_{\mathrm{t}}$, and $k_{\mathrm{t}}$ is integrated in Eq. (2.7) over all accessible values. This means that the resummed anomalous dimension would be needed at all possible values of $\alpha_{s}$ between zero and infinity, which represents a big numerical challenge. In order to avoid this problem, an approximation of the $\alpha_{s}$ dependence of the anomalous dimension was proposed in Ref. [3], where

$$
\begin{aligned}
\gamma_{+}\left(N, \alpha_{s}\left(\mu^{2}\right)\right) & \simeq \frac{\gamma_{+}\left(N, \alpha_{s}\left(\mu_{\mathrm{F}}^{2}\right)\right)}{1+r\left(N, \alpha_{s}\left(\mu_{\mathrm{F}}^{2}\right)\right) \log \left(\mu^{2} / \mu_{\mathrm{F}}^{2}\right)}, \\
r\left(N, \alpha_{s}\right) & =\alpha_{s}^{2} \beta_{0} \frac{d}{d \alpha_{s}} \log \left[\gamma_{+}\left(N, \alpha_{s}\right)\right],
\end{aligned}
$$

with $\beta_{0}$ the one-loop coefficient of the QCD $\beta$-function. Under this assumption, the evolution function becomes

$U\left(N, Q^{2} \xi, \mu_{\mathrm{F}}^{2}\right) \simeq U_{\mathrm{ABF}}\left(N, \frac{Q^{2}}{\mu_{\mathrm{F}}^{2}} \xi\right)$,

having defined

$U_{\mathrm{ABF}}(N, \zeta)=\left(1+r\left(N, \alpha_{s}\right) \log \zeta\right)^{\gamma_{+}\left(N, \alpha_{s}\right) / r\left(N, \alpha_{s}\right)}$. 
Note that $\alpha_{s}$ in Eq. (2.13) is in principle $\alpha_{s}\left(\mu_{\mathrm{F}}^{2}\right)$; however, since the scale dependence of $\alpha_{s}$ is subleading with respect to the leading logarithmic accuracy of the resummed coefficient functions, $\alpha_{s}$ can be computed at any renormalization scale $\mu_{\mathrm{R}}$ without compensating for this change. The name $\mathrm{ABF}$ in Eq. (2.13) comes from the fact that with this approximated evolution function the approach of Refs. $[9,15]$ is recovered, as proven in Ref. [3] for DIS. We will show in the next Sect. 2.3 that this is the case also for processes with two incoming gluons.

A second observation is related to the region of $\xi$ accessible in the integrals Eqs. (2.7) and (2.9). As the strong coupling is running, the integration cannot extend beyond the position of the Landau pole $\Lambda$, identified by the equation

$1+\alpha_{s}\left(\mu^{2}\right) \beta_{0} \log \frac{\Lambda^{2}}{\mu^{2}}=0$,

where $\mu$ is in principle any scale. Solving the equation, we find that the smallest accessible value of $\xi$ is

$\xi_{0}=\frac{\Lambda^{2}}{Q^{2}}=\exp \frac{-1}{\beta_{0} \alpha_{S}\left(Q^{2}\right)}=\frac{\mu_{\mathrm{F}}^{2}}{Q^{2}} \exp \frac{-1}{\beta_{0} \alpha_{s}\left(\mu_{\mathrm{F}}^{2}\right)}$,

where we have written $\xi_{0}$ both in terms of $\mu=Q$ and of $\mu=$ $\mu_{\mathrm{F}}$. In particular, since the approximation Eq. (2.13) assumes $\alpha_{s}$ to be computed at $\mu_{\mathrm{F}}$, the last form is more adequate. Note that when $\xi=\xi_{0}$ the approximate evolution factor reduces to

$$
\begin{aligned}
U\left(N, Q^{2} \xi_{0}, \mu_{\mathrm{F}}^{2}\right) & \simeq U_{\mathrm{ABF}}\left(N, \frac{Q^{2}}{\mu_{\mathrm{F}}^{2}} \xi_{0}\right) \\
& =\left(1-\frac{r\left(N, \alpha_{s}\right)}{\beta_{0} \alpha_{s}}\right)^{\gamma_{+}\left(N, \alpha_{s}\right) / r\left(N, \alpha_{s}\right)},
\end{aligned}
$$

with $\alpha_{s}=\alpha_{s}\left(\mu_{\mathrm{F}}^{2}\right)$. This expression is in general finite; however, from general considerations (see Ref. [4]), we expect the evolution function to vanish in $\xi_{0}$, at least at LL. The vanishing of $U$ in $\xi_{0}$ is a property which turns out to be particularly useful, especially from a numerical point of view. Thus, to force the evolution function to vanish in $\xi=\xi_{0}$, a non-perturbative higher-twist damping function was introduced in Ref. [4],

$D_{\text {higher-twist }}(\xi)= \begin{cases}{\left[1-\left(-\alpha_{s} \beta_{0} \log \xi\right)^{1+\frac{1}{\alpha_{s} \beta_{0}}}\right]} & \xi<1 \\ 1 & \xi>1,\end{cases}$

such that the final approximated expression for the evolution function is

$$
\begin{aligned}
U\left(N, Q^{2} \xi, \mu_{\mathrm{F}}^{2}\right) & \simeq U_{\mathrm{ABF}}^{\mathrm{ht}}\left(N, \frac{Q^{2}}{\mu_{\mathrm{F}}^{2}} \xi\right) \\
& \equiv D_{\text {higher-twist }}\left(\frac{Q^{2}}{\mu_{\mathrm{F}}^{2}} \xi\right) U_{\mathrm{ABF}}\left(N, \frac{Q^{2}}{\mu_{\mathrm{F}}^{2}} \xi\right) .
\end{aligned}
$$

This expression, used throughout this paper, also allows to integrate by parts Eq. (2.7) without producing any boundary term.

Finally, we recall that in Ref. [3] a dedicated anomalous dimension, denoted $\mathrm{LL}^{\prime}$, was constructed specifically for its usage in the evolution function $U$. This $\mathrm{LL}^{\prime}$ anomalous dimension is essentially a LL anomalous dimension, but its dominant small- $x$ singularity is the one of the NLL result. However, in the recent work of Ref. [16] it has been suggested that this hybrid anomalous dimension may give rise to instabilities when expanded in powers of $\alpha_{s}$, as needed for the matching of resummed results to fixed order (we will comment on this in Sect. 2.5). Since the numerical limitations that led to the introduction of the $\mathrm{LL}^{\prime}$ anomalous dimensions have been overcome in Ref. [4], it has thus been proposed in Ref. [16] to use directly the full NLL anomalous dimension, which also corresponds to the original approach of Ref. [15]. In this work we will consider both options later in Sect. 3, and we will provide further support to the suggestion of Ref. [16] of using the NLL anomalous dimension in the evolution function $U$. Thus, the new release of HELL, version 3.0 , performs the resummation using the NLL anomalous dimension in $U$ as default.

To conclude, we report the actual expressions that we will use for the resummation of coefficient functions, as implemented in the code HELL. On top of using the approximated evolution function Eq. (2.18), we integrate by parts so that the derivatives act on the off-shell coefficient function, and we compute the latter in $N=0$, as its $N$ dependence is subleading. The results are

$$
\begin{aligned}
C_{g g}\left(N, \alpha_{s}, \frac{\mu_{\mathrm{F}}^{2}}{Q^{2}}\right)= & \int_{\xi_{0}}^{\infty} d \xi_{1} d \xi_{2} \frac{\partial^{2} \mathcal{C}\left(0, \xi_{1}, \xi_{2}, \alpha_{s}\right)}{\partial \xi_{1} \partial \xi_{2}} \\
& \times U_{\mathrm{ABF}}^{\mathrm{ht}}\left(N, \frac{Q^{2}}{\mu_{\mathrm{F}}^{2}} \xi_{1}\right) U_{\mathrm{ABF}}^{\mathrm{ht}}\left(N, \frac{Q^{2}}{\mu_{\mathrm{F}}^{2}} \xi_{2}\right),
\end{aligned}
$$

$$
\begin{aligned}
C_{\text {aux }}\left(N, \alpha_{s}, \frac{\mu_{\mathrm{F}}^{2}}{Q^{2}}\right)= & \int_{\xi_{0}}^{\infty} d \xi \frac{\partial \mathcal{C}\left(0, \xi, 0, \alpha_{s}\right)}{\partial \xi} \\
& \times U_{\mathrm{ABF}}^{\mathrm{ht}}\left(N, \frac{Q^{2}}{\mu_{\mathrm{F}}^{2}} \xi\right) .
\end{aligned}
$$

The second expression is equivalent to the result in the case of a single hadron in the initial state, such as DIS. The first equation is a new result. The actual numerical implementation of the first equation further requires (for numerical stability) a change of variables, as discussed in Appendix A.1. 


\subsection{Equivalence to the impact factor formulation}

In this section, we show that Eq. (2.19a) leads formally to the same results as the formulation of Ref. [9]. The argument follows closely the one given in Sect. 3.3 of Ref. [3], extending it to the case of two initial gluons. We first introduce the so-called impact factor

$$
\begin{aligned}
\tilde{\mathcal{C}}\left(N, M_{1}, M_{2}, \alpha_{s}, \frac{\mu_{\mathrm{F}}^{2}}{Q^{2}}\right)= & \int d \xi_{1} d \xi_{2} \xi_{1}^{M_{1}} \xi_{2}^{M_{2}} \\
& \times \frac{\partial^{2} \mathcal{C}\left(N, \xi_{1}, \xi_{2}, \alpha_{s}\right)}{\partial \xi_{1} \partial \xi_{2}}\left(\frac{Q^{2}}{\mu_{\mathrm{F}}^{2}}\right)^{M_{1}+M_{2}},
\end{aligned}
$$

which is simply the double Mellin transform with respect to each $k_{\mathrm{t}}^{2}$ of the off-shell coefficient function. For later convenience, we have introduced in the definition of the impact factor a $\mu_{\mathrm{F}}$-dependent term. Because by assumptions there are no collinear singularities, this function is analytic in $M_{1,2}=0$, and thus admits an expansion

$$
\begin{aligned}
& \tilde{\mathcal{C}}\left(N, M_{1}, M_{2}, \alpha_{s}, \frac{\mu_{\mathrm{F}}^{2}}{Q^{2}}\right) \\
& =\sum_{k, j \geq 0} \tilde{\mathcal{C}}_{k j}\left(N, \alpha_{s}, \frac{\mu_{\mathrm{F}}^{2}}{Q^{2}}\right) M_{1}^{k} M_{2}^{j} .
\end{aligned}
$$

Because of the symmetry of the off-shell cross section for the exchange of the virtualities, the coefficients of this expansion are symmetric for the exchange of the indices, $\tilde{\mathcal{C}}_{k j}=\tilde{\mathcal{C}}_{j k}$. Now, we write again the off-shell cross section as the double inverse Mellin transform of Eq. (2.20), expanded as in Eq. (2.21),

$$
\begin{aligned}
\frac{\partial^{2} \mathcal{C}\left(N, \xi_{1}, \xi_{2}, \alpha_{s}\right)}{\partial \xi_{1} \partial \xi_{2}}= & \int \frac{d M_{1}}{2 \pi i} \frac{d M_{2}}{2 \pi i}\left(\frac{Q^{2}}{\mu_{\mathrm{F}}^{2}} \xi_{1}\right)^{-M_{1}} \\
& \times\left(\frac{Q^{2}}{\mu_{\mathrm{F}}^{2}} \xi_{2}\right)^{-M_{2}} \tilde{\mathcal{C}}\left(N, M_{1}, M_{2}, \alpha_{s}\right) \\
= & \sum_{k, j \geq 0} \tilde{\mathcal{C}}_{k j}\left(N, \alpha_{s}, \frac{\mu_{\mathrm{F}}^{2}}{Q^{2}}\right) \\
& \times\left[\partial_{\nu}^{k} \delta\left(v-\log \left(\frac{Q^{2}}{\mu_{\mathrm{F}}^{2}} \xi_{1}\right)\right)\right]_{\nu=0} \\
& \times\left[\partial_{\nu}^{j} \delta\left(v-\log \left(\frac{Q^{2}}{\mu_{\mathrm{F}}^{2}} \xi_{2}\right)\right)\right]_{\nu=0}
\end{aligned}
$$

where we have used the identity

$$
\int \frac{d M}{2 \pi i} \xi^{-M} M^{k}=\left[\partial_{v}^{k} \delta(v-\log \xi)\right]_{v=0}, \quad k \geq 0
$$

We can now plug Eq. (2.22) into Eq. (2.19a) and get ${ }^{3}$

$$
\begin{aligned}
C_{g g} & \left(N, \alpha_{s}, \frac{\mu_{\mathrm{F}}^{2}}{Q^{2}}\right) \\
= & \sum_{k, j \geq 0} \tilde{\mathcal{C}}_{k j}\left(0, \alpha_{s}, \frac{\mu_{\mathrm{F}}^{2}}{Q^{2}}\right)\left[\partial_{\nu}^{k} U_{\mathrm{ABF}}^{\mathrm{ht}}\left(N, e^{\nu}\right)\right]_{\nu=0} \\
& \times\left[\partial_{\nu}^{j} U_{\mathrm{ABF}}^{\mathrm{ht}}\left(N, e^{\nu}\right)\right]_{\nu=0} \cdot
\end{aligned}
$$

This expression, computed at central scale $\mu_{\mathrm{F}}=Q$, reproduces exactly the result of Ref. [9]. Indeed, the derivatives of the evolution function, due to its form Eq. (2.13), satisfy the recursion 4

$$
\begin{aligned}
& {\left[\partial_{v}^{k+1} U_{\mathrm{ABF}}^{\mathrm{ht}}\left(N, e^{v}\right)\right]_{v=0}} \\
& \quad=\left(\gamma_{+}\left(N, \alpha_{s}\right)-k r\left(N, \alpha_{s}\right)\right)\left[\partial_{\nu}^{k} U_{\mathrm{ABF}}^{\mathrm{ht}}\left(N, e^{v}\right)\right]_{\nu=0},
\end{aligned}
$$

which, together with the initial $k=0$ condition $\left[U_{\mathrm{ABF}}^{\mathrm{ht}}\left(N, e^{v}\right)\right]_{\nu=0}=1$, give rise to what is sometimes denoted $\left[\gamma_{+}^{k}\right]$ with squared brackets $[3,15,28]$. In this notation the resummed result is written as

$$
\begin{aligned}
& C_{g g}\left(N, \alpha_{s}, \frac{\mu_{\mathrm{F}}^{2}}{Q^{2}}\right) \\
& =\sum_{k, j \geq 0} \tilde{\mathcal{C}}_{k j}\left(0, \alpha_{s}, \frac{\mu_{\mathrm{F}}^{2}}{Q^{2}}\right)\left[\gamma_{+}^{k}\left(N, \alpha_{s}\right)\right]\left[\gamma_{+}^{j}\left(N, \alpha_{s}\right)\right],
\end{aligned}
$$

which is a straightforward extension of the analogous resummation in the single-hadron case. Note that while using Eq. (2.26) is numerically challenging and necessarily approximate (the infinite series cannot be treated exactly in a numerical code), and its implementation cannot compete with the straightforward integral representation Eq. (2.19a), this form is quite useful for the expansion of the resummed result to fixed order, as we shall now see.

\subsection{Expansion and matching to fixed order}

The resummed results Eq. (2.19), which contains the leading small- $x$ contributions to all orders, are usually matched to a fixed-order contribution. To do so, we need to subtract from the resummed result its expansion in $\alpha_{s}$ up to the fixed-order $k$ considered,

\footnotetext{
${ }^{3}$ Note that the $\mu_{\mathrm{F}}$ dependence is fully included in the coefficients of the expansion. If we hadn't included the $\mu_{\mathrm{F}}$-dependent term in Eq. (2.20), then the $\mu_{\mathrm{F}}$ dependence would be contained in the evolution functions. ${ }^{4}$ Note that the higher-twist term does not play any role in this expansion.
} 


$$
\begin{aligned}
& \Delta_{k} C\left(N, \alpha_{s}, \frac{\mu_{\mathrm{F}}^{2}}{Q^{2}}\right) \\
& =C\left(N, \alpha_{s}, \frac{\mu_{\mathrm{F}}^{2}}{Q^{2}}\right)-\sum_{j=0}^{k} \alpha_{s}^{j} C^{(j)}\left(N, \frac{\mu_{\mathrm{F}}^{2}}{Q^{2}}\right),
\end{aligned}
$$

where the last sum is the truncated $\alpha_{s}$-expansion of the first (resummed) coefficient $C$. Then, this $\Delta_{k} C$ contribution is of $\mathcal{O}\left(\alpha_{s}^{k+1}\right)$, and can be safely added to the fixed $\mathrm{N}^{k} \mathrm{LO}$ result. In this work, we consider the matching up to $\mathrm{N}^{3} \mathrm{LO}$, which is the highest fixed-order accuracy available for Higgs production in gluon fusion. Thus, we need the expansion of the resummation up to $\mathcal{O}\left(\alpha_{s}^{3}\right)$.

The construction of this expansion is obtained in a simple way using the impact-factor formulation, Eq. (2.26). To use it, we first write explicitly $\left[\gamma_{+}^{k}\right]$ up to $k=3$ (omitting the arguments for ease of notation),

$$
\begin{aligned}
& {\left[\gamma_{+}^{0}\right]=1,} \\
& {\left[\gamma_{+}^{1}\right]=\gamma_{+},} \\
& {\left[\gamma_{+}^{2}\right]=\gamma_{+}\left(\gamma_{+}-r\right),} \\
& {\left[\gamma_{+}^{3}\right]=\gamma_{+}\left(\gamma_{+}-r\right)\left(\gamma_{+}-2 r\right),}
\end{aligned}
$$

where $r$ is given in Eq. (2.11). To proceed, we now need to expand in powers of $\alpha_{s}$ both $\gamma_{+}$and $r$. However, before doing so, we recall that in Refs. [3,4] a variant of the resummation, used to estimate the uncertainty from subleading contributions, was introduced in which $r$ is replaced with $\alpha_{s} \beta_{0}$, i.e. the $\alpha_{s}$-dependence of the anomalous dimension is treated as if it was just $\mathcal{O}\left(\alpha_{s}\right)$, in line with the approximation Eq. (2.11). To cover both cases, up to $\mathrm{N}^{3} \mathrm{LO}$ it is sufficient to introduce a single parameter $T$, which equals 2 in the default case, and equals 1 in the limit $r=\alpha_{s} \beta_{0}$. Introducing the expansion of the anomalous dimension

$\gamma_{+}=\alpha_{s} \gamma_{0}+\alpha_{s}^{2} \gamma_{1}+\alpha_{s}^{3} \gamma_{2}+\mathcal{O}\left(\alpha_{s}^{4}\right)$

we can indeed write

$r=\alpha_{s} \beta_{0}\left[1+(T-1) \alpha_{s} \frac{\gamma_{1}}{\gamma_{0}}+\mathcal{O}\left(\alpha_{s}^{2}\right)\right]$.

With these expressions we can expand Eq. (2.28) as

$$
\begin{aligned}
& {\left[\gamma_{+}^{0}\right]=1} \\
& {\left[\gamma_{+}^{1}\right]=\alpha_{s} \gamma_{0}+\alpha_{s}^{2} \gamma_{1}+\alpha_{s}^{3} \gamma_{2}+\mathcal{O}\left(\alpha_{s}^{4}\right)} \\
& {\left[\gamma_{+}^{2}\right]=\alpha_{s}^{2} \gamma_{0}\left(\gamma_{0}-\beta_{0}\right)+\alpha_{s}^{3} \gamma_{1}\left(2 \gamma_{0}-T \beta_{0}\right)+\mathcal{O}\left(\alpha_{s}^{4}\right)} \\
& {\left[\gamma_{+}^{3}\right]=\alpha_{s}^{3} \gamma_{0}\left(\gamma_{0}-\beta_{0}\right)\left(\gamma_{0}-2 \beta_{0}\right)+\mathcal{O}\left(\alpha_{s}^{4}\right)}
\end{aligned}
$$

which can be now used in Eq. (2.26) to get the $\alpha_{S}$-expansion of the $g g$ coefficient function:

$$
\begin{aligned}
C_{g g}= & \tilde{\mathcal{C}}_{00}+\alpha_{s} 2 \tilde{\mathcal{C}}_{10} \gamma_{0} \\
& +\alpha_{s}^{2}\left[2 \tilde{\mathcal{C}}_{10} \gamma_{1}+2 \tilde{\mathcal{C}}_{20} \gamma_{0}\left(\gamma_{0}-\beta_{0}\right)+\tilde{\mathcal{C}}_{11} \gamma_{0}^{2}\right] \\
& +\alpha_{s}^{3}\left[2 \tilde{\mathcal{C}}_{10} \gamma_{2}+2 \tilde{\mathcal{C}}_{20} \gamma_{1}\left(2 \gamma_{0}-T \beta_{0}\right)\right. \\
& +2 \tilde{\mathcal{C}}_{11} \gamma_{0} \gamma_{1}+2 \tilde{\mathcal{C}}_{30} \gamma_{0}\left(\gamma_{0}-\beta_{0}\right)\left(\gamma_{0}-2 \beta_{0}\right) \\
& \left.+2 \tilde{\mathcal{C}}_{21} \gamma_{0}^{2}\left(\gamma_{0}-\beta_{0}\right)\right]+\mathcal{O}\left(\alpha_{s}^{4}\right) .
\end{aligned}
$$

Depending on the anomalous dimension used in the evolution function $U$ (see discussion in Sect. 2.2), which determines the actual form of $\gamma_{0,1,2}$, this expression provides the first few orders of the resummed coefficient function needed to construct the resummed contribution $\Delta_{k} C_{g g}$ up to $k=3$.

To construct the expansion of the resummed coefficient functions for the other partonic channels, we need to expand the auxiliary function Eq. (2.19b). Straightforwardly, its impact-factor form can be derived from Eq. (2.26) by keeping only the $j=0$ part of the sum, and flipping the sign

$C_{\text {aux }}\left(N, \alpha_{s}, \frac{\mu_{\mathrm{F}}^{2}}{Q^{2}}\right)=-\sum_{k \geq 0} \tilde{\mathcal{C}}_{k 0}\left(0, \alpha_{s}, \frac{\mu_{\mathrm{F}}^{2}}{Q^{2}}\right)\left[\gamma_{+}^{k}\left(N, \alpha_{s}\right)\right]$.

Thus, its $\alpha_{s}$ expansion is given by

$$
\begin{aligned}
C_{\mathrm{aux}}= & -\tilde{\mathcal{C}}_{00}-\alpha_{s} \tilde{\mathcal{C}}_{10} \gamma_{0}-\alpha_{s}^{2}\left[\tilde{\mathcal{C}}_{10} \gamma_{1}+\tilde{\mathcal{C}}_{20} \gamma_{0}\left(\gamma_{0}-\beta_{0}\right)\right] \\
& -\alpha_{s}^{3}\left[\tilde{\mathcal{C}}_{10} \gamma_{2}+\tilde{\mathcal{C}}_{20} \gamma_{1}\left(2 \gamma_{0}-T \beta_{0}\right)\right. \\
& \left.+\tilde{\mathcal{C}}_{30} \gamma_{0}\left(\gamma_{0}-\beta_{0}\right)\left(\gamma_{0}-2 \beta_{0}\right)\right]+\mathcal{O}\left(\alpha_{s}^{4}\right)
\end{aligned}
$$

Form Eqs. (2.32) and (2.34) we can construct the expansions of the quark coefficient functions, according to Eq. (2.10),

$$
\begin{aligned}
C_{q g}= & \frac{C_{F}}{C_{A}}\left[\alpha_{s} \tilde{\mathcal{C}}_{10} \gamma_{0}+\alpha_{s}^{2}\left[\tilde{\mathcal{C}}_{10} \gamma_{1}+\tilde{\mathcal{C}}_{20} \gamma_{0}\left(\gamma_{0}-\beta_{0}\right)+\tilde{\mathcal{C}}_{11} \gamma_{0}^{2}\right]\right. \\
& +\alpha_{s}^{3}\left[\tilde{\mathcal{C}}_{10} \gamma_{2}+\tilde{\mathcal{C}}_{20} \gamma_{1}\left(2 \gamma_{0}-T \beta_{0}\right)+2 \tilde{\mathcal{C}}_{11} \gamma_{0} \gamma_{1}\right. \\
& \left.\left.+\tilde{\mathcal{C}}_{30} \gamma_{0}\left(\gamma_{0}-\beta_{0}\right)\left(\gamma_{0}-2 \beta_{0}\right)+2 \tilde{\mathcal{C}}_{21} \gamma_{0}^{2}\left(\gamma_{0}-\beta_{0}\right)\right]+\mathcal{O}\left(\alpha_{s}^{4}\right)\right], \\
C_{q q}= & \left(\frac{C_{F}}{C_{A}}\right)^{2}\left[\alpha_{s}^{2} \tilde{\mathcal{C}}_{11} \gamma_{0}^{2}+\alpha_{s}^{3}\left[2 \tilde{\mathcal{C}}_{11} \gamma_{0} \gamma_{1}\right.\right. \\
& \left.\left.+2 \tilde{\mathcal{C}}_{21} \gamma_{0}^{2}\left(\gamma_{0}-\beta_{0}\right)\right]+\mathcal{O}\left(\alpha_{s}^{4}\right)\right] .
\end{aligned}
$$

With these expressions it is then possible to construct also the resummed contributions $\Delta_{k} C_{q g}$ and $\Delta_{k} C_{q q}$ for the quark coefficient functions up to $k=3$. All together, these expressions allow to match resummed results to $\mathrm{N}^{3} \mathrm{LO}$. The computation of the $\tilde{\mathcal{C}}_{i j}$ coefficients, needed for the expansions presented here, is detailed in Appendix A.2. 
2.5 The first few orders of the anomalous dimension at $\mathrm{LL}^{\prime}$ and NLL

To conclude the section, we now present the analytic expressions of the $\mathcal{O}\left(\alpha_{S}^{1,2,3}\right)$ anomalous dimensions $\gamma_{0,1,2}$ needed for the matching of the resummed coefficient function to fixed order up to $\mathrm{N}^{3}$ LO, Eqs. (2.32), (2.35) and (2.36). We treat both the case in which the anomalous dimension used is the LL' introduced in Ref. [3] and the case in which the full NLL anomalous dimension is used, as suggested in Refs. [15, 16], see discussion in Sect. 2.2. These expressions are obtained by expanding the purely resummed LL' or NLL anomalous dimension, and have been already computed and presented in Refs. $[3,4,16]$. Thus, here we only report the final results [16]. For LL' resummation we have

$$
\begin{aligned}
\gamma_{0}^{\mathrm{LL}}= & \frac{a_{11}}{N}+\frac{a_{10}}{N+1}, \\
\gamma_{1}^{\mathrm{LL}}= & \beta_{0} a_{11}\left(\frac{21}{8} \zeta_{3}-4 \log 2\right)\left(\frac{1}{N}-\frac{4 N}{(N+1)^{2}}\right) \\
\gamma_{2}^{\mathrm{LL}}= & \frac{\lambda_{2}}{N^{2}}+\frac{\lambda_{1}}{N}-\left(\lambda_{2}+\lambda_{1}\right) \frac{4 N}{(N+1)^{2}} \\
& +\left(\frac{a_{11}}{N^{2}}+\frac{2\left(a_{11}+a_{10}\right)}{(N+1)^{2}}\right) \\
& \times\left[\frac{a_{11} a_{10}}{(1+N)^{2}}-\frac{a_{11} a_{10}}{4} \frac{4 N}{(N+1)^{2}}\right. \\
& +a_{11}\left(\frac{a_{11}}{N}+a_{10}-\frac{2\left(a_{11}+a_{10}\right) N}{N+1}\right) \\
& \left.\times\left[\psi_{1}(1+N)-\zeta_{2}\right]\right]
\end{aligned}
$$

while for NLL resummation the results are

$$
\begin{aligned}
\gamma_{0}^{\mathrm{NLL}}= & \frac{a_{11}}{N}+\frac{a_{10}}{N+1}, \\
\gamma_{1}^{\mathrm{NLL}}= & \frac{a_{21}}{N}-\frac{2 a_{21}}{N+1}, \\
\gamma_{2}^{\mathrm{NLL}}= & \frac{7}{4} \beta_{0}^{2} a_{11} \zeta_{3}\left(\frac{1}{N}-\frac{4 N}{(N+1)^{2}}\right) \\
& +\left(\frac{a_{11}}{N^{2}}+\frac{2\left(a_{11}+a_{10}\right)}{(N+1)^{2}}\right) \\
& \times\left[\rho+\frac{a_{21}}{1+N}+\frac{a_{11} a_{10}}{(1+N)^{2}}\right. \\
& -\left(\rho+\frac{a_{21}}{2}+\frac{a_{11} a_{10}}{4}-\beta_{0} a_{11}\right) \frac{4 N}{(N+1)^{2}} \\
& +a_{11}\left(\frac{a_{11}}{N}+a_{10}-\frac{2\left(a_{11}+a_{10}\right) N}{N+1}+\beta_{0}\right) \\
& \left.\times\left[\psi_{1}(1+N)-\zeta_{2}\right]\right] .
\end{aligned}
$$

The coefficients appearing above are

$a_{11}=\frac{C_{A}}{\pi}$,

$a_{21}=n_{f} \frac{26 C_{F}-23 C_{A}}{36 \pi^{2}}$,

$a_{10}=-\frac{11 C_{A}+2 n_{f}\left(1-2 C_{F} / C_{A}\right)}{12 \pi}$,

and

$$
\begin{aligned}
\lambda_{2}= & 1.26717+0.110072 n_{f}, \\
\lambda_{1}= & \left\{\begin{array}{l}
-60.6782+3.53857 n_{f}+0.00841828 n_{f}^{2} \\
-30.3568+1.77143 n_{f}+0.00414421 n_{f}^{2}
\end{array}\right. \\
\rho= & \frac{1}{\pi^{2}}\left[C_{A}^{2}\left(-\frac{74}{27}+\frac{11}{12} \zeta_{2}+\frac{5}{2} \zeta_{3}\right)\right. \\
& \left.+n_{f} C_{A}\left(\frac{4}{27}+\frac{1}{6} \zeta_{2}\right)+n_{f} C_{F}\left(\frac{7}{27}-\frac{1}{3} \zeta_{2}\right)\right] .
\end{aligned}
$$

The two values of $\lambda_{1}$, Eq. (2.44b), come from another variant of the resummation, used in the construction of $\gamma_{+}$, which affects only the $\mathrm{LL}^{\prime}$ anomalous dimension at this order. More details can be found in Appendix A of Ref. [16]. All these expressions are implemented in HELL 3.0.

Before moving on, we would like to comment on a particular feature of these expansions. We recall that, due to accidental cancellations, the expected leading singularities of the NLO and NNLO anomalous dimensions are zero. Since both $\mathrm{LL}^{\prime}$ and NLL anomalous dimension are accurate at LL, the leading terms $1 / N^{2}$ in $\gamma_{1}$ and $1 / N^{3}$ in $\gamma_{2}$ are correctly absent. As a consequence, the highest singularity in $\gamma_{1}$ and $\gamma_{2}$ is the NLL one, which is correct only in the NLL anomalous dimension. Instead, the dominant singularity (and any other subleading term) of these two orders in the $\mathrm{LL}^{\prime}$ result is not correct. Thus, while the all-order LL' and NLL anomalous dimensions may be in good agreement (and indeed they are), their $\mathcal{O}\left(\alpha_{s}^{2}\right)$ and $\mathcal{O}\left(\alpha_{s}^{3}\right)$ expansions may be very different (and indeed they differ substantially at $\mathcal{O}\left(\alpha_{s}^{3}\right)$ ). For this reason, resummed results which depend explicitly on $\gamma_{1}^{\mathrm{LL}^{\prime}}$ and $\gamma_{2}^{\mathrm{LL}^{\prime}}$ (such as resummed results matched to NNLO and beyond) may differ substantially from those obtained with the NLL anomalous dimension, and when this is the case results obtained in the NLL case have to be favoured. It has been noticed in Ref. [16] (and we will see it also here in Sect. 3) that when matching to $\mathrm{N}^{3} \mathrm{LO}$ resummed results based on $\mathrm{LL}^{\prime}$ behave pathologically at medium/large values of $x$, which is a consequence of a similar behaviour in the inverse Mellin transform of $\gamma_{2}^{L^{\prime}}$. This is the main motivation that induced Ref. [16] to propose the use of the NLL anomalous dimension as default. 


\section{Resummed Higgs cross section at the $\mathrm{LHC}$ and beyond}

We now turn our attention to a hadron-hadron collider process which is of great interest for LHC phenomenology: Higgs production in gluon fusion ( $\mathrm{ggH}$ for short). Of course, Higgs physics is very interesting because the Higgs sector can be sensitive to new physics beyond the Standard Model. The inclusive Higgs cross section, which we are going to consider, is for instance sensitive to heavy particles coupling to gluons, which may then run in the loop of Fig. 1 and alter the production rate at the LHC.

Moreover, from a theoretical point of view, Higgs production is an interesting process because fixed-order perturbative QCD corrections are very large, with NLO being about twice the LO, and NNLO adding another $\sim 40 \%$ of the LO to the cross section. This motivated various studies to go beyond NNLO [28-34], culminating in the computation of this production process to $\mathrm{N}^{3} \mathrm{LO}[5-8]$ in the large top-mass limit. ${ }^{5}$ It has been demonstrated in various ways that such large corrections mostly originate from softvirtual contributions $[28,30,33,34,38]$, dominant at large $x$, and can be resummed to all orders by means of threshold resummation techniques [29,39-41], reaching $\mathrm{N}^{3} \mathrm{LL}$ accuracy $[42-46] .{ }^{6} \mathrm{~N}^{3} \mathrm{LO}+\mathrm{N}^{3} \mathrm{LL}$ predictions are very close to $\mathrm{N}^{3} \mathrm{LO}$ ones, thus suggesting that perturbative expansion is apparently converging, and giving some confidence that current theoretical predictions, such as the one recommended by the LHC Higgs Cross Section Working Group (HXSWG) [51], are sufficiently accurate for precision phenomenology.

In this work we investigate the effect of the all-order resummation of the small- $x$ logarithms, i.e. those important in the opposite limit with respect to the one largely studied for this process. Indeed, Higgs production in gluon fusion is also one of the LHC processes which is expected to be most sensitive to small- $x$ logarithmic enhancement, due to the fact that it is gluon-gluon initiated at lowest order, and the gluon PDF is the most sensitive to small- $x$ resummation effects. We will show that the consistent inclusion of small- $x$ resummation has a sizeable effect. In the $Q_{0} \overline{\mathrm{MS}}$ scheme that we adopt, most of this effect comes from the use of resummed PDF instead of fixed-order ones, while the effect of resummation in the coefficient function is much milder. The effect of resummation gets progressively larger as the collider energy

\footnotetext{
$\overline{5 \text { A consistent }} \mathrm{N}^{3} \mathrm{LO}$ calculation would also require PDFs fitted and evolved with $\mathrm{N}^{3} \mathrm{LO}$ theory. However, the four-loop DGLAP splitting functions are not yet fully known, though recently there has been impressive progress towards their computation [35-37]. Thus, at the moment all $\mathrm{N}^{3} \mathrm{LO}$ predictions use NNLO PDFs.

6 To be precise, all contributions that are relevant for $\mathrm{N}^{3} \mathrm{LL}$ are known $[29,31,47,48]$, with the exception of the four-loop cusp anomalous dimension (see $[49,50]$ for recent progress), which is thought to have a negligible impact.
}

is increased, since smaller and smaller values of $x$ become accessible and increasingly important. Therefore, the inclusion of resummation becomes more crucial at higher-energy colliders, such as the high-energy phase of the LHC, with $\sqrt{s}=27 \mathrm{TeV}$, and even more at a future circular hadron collider (FCC-hh) of $\sqrt{s}=100 \mathrm{TeV}$.

Before presenting resummed results, we recall that many results in the computation of the Higgs production cross section in gluon fusion are obtained within the so-called large top-mass effective field theory (EFT henceforth), where the top-quark is integrated out of the theory and its effect included as corrections in powers of $m_{H}^{2} / m_{t}^{2}$. However, in this theory the small- $x$ region cannot be predicted correctly, as the $x \rightarrow 0$ limit does not commute with the $m_{t} \rightarrow \infty$ limit of the EFT. Therefore, the correct inclusion of small- $x$ resummation also requires a correct treatment of the small- $x$ region at fixed order. In Sect. 3.1, we then first revisit how top mass dependence is included in fixed-order result and how the correct small $-x$ logarithms can be included at fixed order. Then, in Sects. 3.2 and 3.3 we will show the impact of small- $x$ resummation at parton level and on the cross section, respectively.

This section provides a detailed explanation of the small$x$ resummed results presented in Ref. [17] in the context of a double small- $x$ plus large- $x$ resummation.

\subsection{Construction of the fixed-order result at small $x$ with top mass dependence}

The LO diagram for $g g H$ production, Fig. 1, is a one-loop diagram with a massive internal particle. The NLO correction to this process has been carried out exactly $[52,53]$. However, from NNLO onward, the exact computation would require the evaluation of three-loop (or higher) diagrams with massive internal lines, which are out of reach of the current computational technology.

However, in the limit in which the partonic center-of-mass energy $\sqrt{\hat{s}}$ is (much) smaller than twice the top mass $m_{t}$, one can construct an effective field theory (EFT) in which the top loop shrinks to a point, leading to a pointlike interaction described by operators. The operator with the lower dimensionality does not depend explicitly on the top mass, except for a logarithmic dependence appearing in its Wilson coefficient. Operators with higher dimensionality will give rise to corrections suppressed by increasing powers of $1 / \mathrm{m}^{2}$.

Within this EFT it has been possible to push the computation of the $g g H$ cross section at NNLO (both at the leading power level [54-56] and including a few corrections in $1 / m_{t}^{2}[27,57,58]$ ) and even at $\mathrm{N}^{3} \mathrm{LO}[5-8]$ (at leading power). Because the expansion parameter of the EFT is

$$
\frac{\hat{s}}{4 m_{t}^{2}}=\frac{m_{H}^{2}}{4 z m_{t}^{2}},
$$


where $z=m_{H}^{2} / \hat{s}$ and $\hat{s}$ the partonic center-of-mass energy, the limits $m_{t} \rightarrow \infty$ and $z \rightarrow 0$ do not commute. ${ }^{7}$ Thus, the EFT cannot describe the small- $z$ region correctly. For this reason, computations within the EFT can be (and have been) carried out as threshold expansions about $z=1$, i.e. as power series in $(1-z)$, e.g. in Refs. [7,57]. Indeed, at large and medium $z$ this expansion converges to the exact result, while at small $z$ it is wrong anyway.

The goal of this subsection is to provide a way to supplement computations in the EFT with the exact small- $z$ logarithms, which can be predicted from the resummation. Let's consider the generic coefficient function with perturbative expansion (omitting all unnecessary arguments and indices for simplicity)

$C\left(z, \alpha_{s}, \frac{m_{H}^{2}}{m_{t}^{2}}\right)=\sum_{k=0}^{\infty} \alpha_{s}^{k} C^{(k)}\left(z, \frac{m_{H}^{2}}{m_{t}^{2}}\right)$.

As we already stated, from NNLO onwards the exact $m_{H} / m_{t}$ dependence is unknown. At NNLO, $m_{H} / m_{t}$ effects have been computed as an expansion in

$\rho_{t}=\frac{m_{H}^{2}}{m_{t}^{2}}$

up to the order $p_{\max }=3[27,57,58]$,

$C^{(2)}\left(z, \rho_{t}\right) \simeq \sum_{p=0}^{p_{\max }} \rho_{t}^{p} C_{p}^{(2)}(z)$

while at $\mathrm{N}^{3} \mathrm{LO}$ only the first term is known $\left(p_{\max }=0\right)$ [5-8]. However, the expansion in $m_{H} / m_{t}$ is not accurate at small $z$, since the actual expansion parameter is the one given in Eq. (3.1): in particular, the $\rho_{t}$ expansion is supposed to break down for $z \lesssim \rho_{t} / 4$. Therefore, the small- $z$ behaviour of the $m_{H} / m_{t}$ expansion is unstable,

$$
\begin{aligned}
C^{(k)}\left(z, \rho_{t}\right)= & \sum_{p=0}^{p_{\max }} \rho_{t}^{p} \sum_{j=0}^{p} \sum_{n=0}^{2 k-1} B_{p, j, n}^{(k)} \frac{\log ^{n} z}{z^{1+j}} \\
& +\mathcal{O}\left(z^{0}\right)+\mathcal{O}\left(\rho_{t}^{p_{\max }+1}\right),
\end{aligned}
$$

exhibiting double-logarithmic enhancement and higher powers of $1 / z$ at each extra order in $\rho_{t}$, in contrast with the exact small- $z$ behaviour

$C^{(k)}\left(z, \rho_{t}\right)=\sum_{n=0}^{k-1} A_{n}^{(k)}\left(\rho_{t}\right) \frac{\log ^{n} z}{z}+\mathcal{O}\left(z^{0}\right)$,

\footnotetext{
${ }^{7}$ Note that the variable $z$ is a scaling variable as $x$ in DIS and in the PDFs, and thus small- $x$ resummation in the Higgs partonic coefficient functions resums logarithms of $z$.
}

which is single-logarithmic enhanced and always contains a single power of $1 / z$. The exact small- $z$ behaviour, Eq. (3.6), can be predicted (at least at LL, i.e. $n=k-1$ ) from highenergy resummation. Our goal is therefore to understand how the exact Eq. (3.6) can be used to replace the wrong Eq. (3.5) of the large $m_{t}$ EFT computation. We recall that two different phenomenological solutions to this problem have been already proposed in Refs. $[27,57,58]$. Here, we want to deal with this problem in a systematic way.

As a first step, we need to understand whether the limit $p_{\max } \rightarrow \infty$ converges to the exact result or not. At large $z$ and for sufficiently small $\rho_{t}$, the answer must be yes, or at least asymptotically yes. On the other hand, at small $z$ the expansion clearly diverges, with new singularities appearing at each order in $\rho_{t}$, Eq. (3.5). Thus, at small $z$, only the all-order sum may make sense, but certainly not any finite truncation of it. Therefore, in order to build up a sensible result, we need to make sure first to get rid of the bad small$z$ behaviour of the $\rho_{t}$ expansion, and once this is done we can add the exact small- $z$ contribution, Eq. (3.6). The final expression must be such that the limit $p_{\max } \rightarrow \infty$ tends to the exact result. ${ }^{8}$ We will consider four possible approaches, in turn.

Method of subtraction The first option that we consider consists in subtracting from the $\rho_{t}$ expansion the "wrong" small$z$ behaviour, Eq. (3.5), replacing it with the exact small $z$, Eq. (3.6). The resulting expression is

$$
\begin{aligned}
C^{(k)}\left(z, \rho_{t}\right) \simeq & \sum_{p=0}^{p_{\max }} \rho_{t}^{p}\left[C_{p}^{(k)}(z)-d(z) \sum_{j=0}^{p} \sum_{n=0}^{2 k-1} B_{p, j, n}^{(k)} \frac{\log ^{n} z}{z^{1+j}}\right] \\
& +d(z) \sum_{n=0}^{k-1} A_{n}^{(k)}\left(\rho_{t}\right) \frac{\log ^{n} z}{z}
\end{aligned}
$$

where we have further introduced a function $d(z)$, which represents a possible large- $z$ damping to be uniformly applied to the small- $z$ parts of the result. The role of this damping is to suppress the effect of the small- $z$ contributions at large $z$ : indeed, the $1 / z$ terms without logarithms contained in the small $z$ contributions do not vanish at large $z$.

This method, despite its simplicity and naturalness, has three important drawbacks. The first is that it requires the exact EFT result, and not just its (simpler to compute) threshold expansion, which, as we already mentioned, carries the same correct information, and differs only in the region where they are both wrong. At NNLO, the EFT small- $z$ contribution is fully known for $p=0,1,2$ [58], while the threshold expansion is also known for $p=3[27,57]$. At $\mathrm{N}^{3} \mathrm{LO}$, only the leading term $B_{0,0,5}^{(3)}$ was known until very recently, when

\footnotetext{
8 Possibly up to subleading power logarithmic contributions behaving as $\log z$ without any $1 / z$ enhancement.
} 
in Ref. [8] the exact leading EFT result ( $p=0$ ) has been computed, thus allowing to use this method at $\mathrm{N}^{3} \mathrm{LO}$ as well. The second is that the function in squared brackets in Eq. (3.7) still contains double-logarithmic terms at $\mathcal{O}\left(z^{0}\right)$ which are not predicted correctly by the EFT expansion either, and can thus potentially contaminate the result. (In principle these logarithmic contributions could be subtracted as well, however their counterparts in the exact theory cannot be derived from small- $z$ resummation and thus they cannot be added back.) The third and perhaps more severe issue is that the result may strongly depend on the damping function used. Indeed, ideally, the two small- $z$ contributions should cancel each other at large $z$. However, since the $z \rightarrow 1$ limit of the small- $z$ contribution in the $\rho_{t}$ expansion inherits its instability, there is no practical compensation at large $z$ between what is subtracted and what is added. And this must not happen, since the $C_{p}^{(k)}(z)$ terms are supposed to be reliable in the $z \rightarrow 1$ limit. Thus the damping becomes a necessity, but its form is not prescribed by the procedure, leaving a degree of arbitrariness which may contaminate the result.

Method of threshold expansion The expression in square brackets in Eq. (3.7) does no longer contain divergent terms at small $z .{ }^{9}$ Thus, there is no loss of information if it is replaced with its threshold expansion, i.e. an expansion in powers of $(1-z)$. Let us define

$\delta C_{p}^{(k)}(z)=C_{p}^{(k)}(z)-d(z) \sum_{j=0}^{p} \sum_{n=0}^{2 k-1} B_{p, j, n}^{(k)} \frac{\log ^{n} z}{z^{1+j}}$

to be the function in square brackets in Eq. (3.7). Equation (3.8) can be expanded at large $z$ as ${ }^{10}$

$$
\begin{aligned}
\delta C_{p}^{(k)}(z) & =z^{a}\left[\frac{\delta C_{p}^{(k)}(z)}{z^{a}}\right]_{\text {t.e. }} \\
& =z^{a} \sum_{i=0}^{\infty}(1-z)^{i}\left[c_{p, i}^{(k)}(a, \ell)-\sum_{j=0}^{p} \sum_{n=0}^{2 k-1} B_{p, j, n}^{(k)} b_{j, n, i}(a)\right],
\end{aligned}
$$

where $a$ is a parameter, and the subscript "t.e." (threshold expansion) means that the function enclosed by those brackets is expanded in powers of $1-z$. In the equation above, the expansion coefficients $c_{p, i}^{(k)}$ also depend in general on

$\ell \equiv \log (1-z)$

\footnotetext{
${ }^{9}$ Except for the aforementioned powers of $\log z$ without $1 / z$ enhancement, which are not predicted correctly either.

10 To simplify the following discussion, let us assume that for the $g g$ channel the coefficient function is defined as the "regular" part of the decomposition $C_{g g}^{(k)}=\left[C_{g g}^{(k)}\right]_{\text {distr }}+\left[C_{g g}^{(k)}\right]_{\text {reg }}$, where the distributional part contains plus distributions and $\delta(1-z)$ functions, and the regular part everything else.
}

which is clearly not expandable in $z=1$, and we have introduced the coefficients $b_{j, n, i}(a)$ according to

$d(z) \frac{\log ^{n} z}{z^{1+j+a}}=\sum_{i=0}^{\infty}(1-z)^{i} b_{j, n, i}(a)$,

which thus depend on the damping function $d(z)$. The $a$ parameter is in principle free, since the result is independent of $a$ when the whole series in $1-z$ is considered. Of course, any finite truncation of the series to $i=i_{\max }$ will have a residual dependence on $a$, which can thus be used e.g. to estimate the uncertainty due to missing terms in the threshold expansion [7]. The coefficients $C_{p}^{(k)}(z)$ have been computed in the first place as a threshold expansion at NNLO $[27,57]$ and $\mathrm{N}^{3} \mathrm{LO}$ [7], so the relevant $c_{p, i}^{(k)}$ are all known.

Let us comment on the choice of the parameter $a$. The value $a=-1$ is the one adopted in Refs. [27,57] (as there the partonic cross section $z C(z)$ is expanded). ${ }^{11}$ This choice is such that terms behaving as $1 / z$ are generated in the threshold expansion; however, these terms are not predicted correctly by the EFT, and indeed they have been subtracted in Eq. (3.8), so producing them can be dangerous. On the contrary, we note that if we choose $a \geq 0$ both terms in Eq. (3.8) lead to a threshold expansion which does not grow at small $z$. In particular, for $a=0$ the threshold expansion goes to a constant, while for larger $a$ it vanishes in $z=0$ (however $a$ cannot be too large, otherwise it would affect the coefficient function in a region of medium $z$ where the threshold expansion is reliable). This means that choosing $a \geq 0$ the resulting coefficient function does not contain any leading small- $z$ contribution. Thus, the threshold expansion with $a \geq 0$ provides a natural and legitimate way of damping the EFT result at small- $z$, thereby also removing the potential danger induced by the EFT logarithmic terms at $\mathcal{O}\left(z^{0}\right)$.

This observation may suggest that, as long as the coefficient function is threshold-expanded with $a \geq 0$, the term $\delta C_{p}^{(k)}(z)$, Eq. (3.8), appearing in Eq. (3.7) can be replaced with just the full coefficient function $C_{p}^{(k)}(z)$, without subtracting the small- $z$ terms. Indeed, at large $z$ the two objects do not differ, due to the damping $d(z)$ which suppresses the subtraction terms, and at small $z$ both objects do not contain small- $z$ contributions. Clearly, the subleading small- $z$ terms (those not enhanced by $1 / z$ ) may differ, but these are anyway beyond our control, and certainly not predicted by the last term of Eq. (3.7). Thus, we may conclude that an equally good definition of the full result is

\footnotetext{
$\overline{11}$ To be precise, in Refs. [27,57] also the distributional part in the $g g$ channel is multiplied by $1 / z$, thus changing the actual $c_{p, i}^{(k)}$ coefficients. However, this difference is immaterial for the present discussion.
} 


$$
\begin{aligned}
C^{(k)}\left(z, \rho_{t}\right) \simeq & z^{a} \sum_{p=0}^{p_{\max }} \rho_{t}^{p} \sum_{i=0}^{i_{\max }}(1-z)^{i} c_{p, i}^{(k)}(a, \ell) \\
& +d(z) \sum_{n=0}^{k-1} A_{n}^{(k)}\left(\rho_{t}\right) \frac{\log ^{n} z}{z},
\end{aligned}
$$

provided $a \geq 0$. In fact, Eq. (3.12) can be obtained with no approximations, by exploiting the dependence on the damping function $d(z)$. Indeed, in this case, the damping function is no longer fully free, but we have a guiding principle how to choose its form. Namely, since at large $z$ the first part of Eq. (3.12) is reliable up to $\mathcal{O}\left((1-z)^{i_{\max }}\right)$, the damping function must be suppressed at least as

$d(z)=(1-z)^{i_{\max }+1}$,

such that the exact small- $z$ part does not spoil the accuracy of the threshold expansion. With this choice for $d(z)$, the $b_{j, n, i}(a)$ coefficients are all zero for $i \leq i_{\max }$ : hence, up to $i=i_{\max }$, the EFT small- $z$ terms do vanish. Thus, when using this damping function (or a more suppressed version of it), the threshold expansion of Eq. (3.7) gives exactly Eq. (3.12). Note that, because in Eq. (3.12) there is no subtraction of small- $z$ EFT contributions, this formulation is simpler and very suitable for numerical implementation, both at NNLO and at $\mathrm{N}^{3} \mathrm{LO}$.

Method of double subtraction In Refs. [27,57] a different construction was considered, where the exact small $z$ is added to the threshold expansion of $C_{p}^{(k)}(z)$ after having subtracted from it its own threshold expansion, without applying any damping. To derive this possible approach, let us start again from Eq. (3.7), to which we replace the first part with its threshold expansion, and where we add and subtract the threshold expansion of the exact small $z$,

$$
\begin{aligned}
C^{(k)}\left(z, \rho_{t}\right) \simeq & z^{a} \sum_{i=0}^{i_{\max }}(1-z)^{i} \\
& \times\left[\sum_{p=0}^{p_{\max }} \rho_{t}^{p}\left(c_{p, i}^{(k)}(a, \ell)-\sum_{j=0}^{p} \sum_{n=0}^{2 k-1} B_{p, j, n}^{(k)} b_{j, n, i}(a)\right)\right. \\
& \left.+\sum_{n=0}^{k-1} A_{n}^{(k)}\left(\rho_{t}\right) b_{0, n, i}(a)\right]+\sum_{n=0}^{k-1} A_{n}^{(k)}\left(\rho_{t}\right) \\
& \times\left[d(z) \frac{\log ^{n} z}{z}-z^{a} \sum_{i=0}^{i_{\max }}(1-z)^{i} b_{0, n, i}(a)\right] .
\end{aligned}
$$

As far as the exact small- $z$ term is concerned, it is clear that the damping function becomes unnecessary with this approach, as the term in square brackets in the last line of Eq. (3.14) is of $\mathcal{O}\left((1-z)^{i_{\max }+1}\right)$ irrespectively of the form of $d(z)$. If we choose the damping function as in Eq. (3.13) then we recover exactly Eq. (3.12), since $b_{j, n, i}(a)=0$ for $i \leq i_{\max }$. However, following Refs. [27,57], we can now choose

$d(z)=1$,

thus fixing the form of the $b_{j, n, i}(a)$ coefficients according to Eq. (3.11). The approach of Refs. [27,57] is obtained by ignoring the second term of the second line and the first term of the third line of the first line of Eq. (3.14), such that the final result is

$$
\begin{aligned}
C^{(k)}\left(z, \rho_{t}\right) \simeq & z^{a} \sum_{p=0}^{p_{\max }} \rho_{t}^{p} \sum_{i=0}^{i_{\max }}(1-z)^{i} c_{p, i}^{(k)}(a, \ell) \\
& +\sum_{n=0}^{k-1} A_{n}^{(k)}\left(\rho_{t}\right)\left[\frac{\log ^{n} z}{z}-z^{a} \sum_{i=0}^{i_{\max }}(1-z)^{i} b_{0, n, i}(a)\right] .
\end{aligned}
$$

We notice immediately that this form is very similar to Eq. (3.12), with the difference that the large- $z$ behaviour of the exact small- $z$ contribution is subtracted rather than being damped. The result is in both cases a small- $z$ contribution which starts at $\mathcal{O}\left((1-z)^{i_{\max }+1}\right)$, and with the same small- $z$ behaviour: it should thus give similar results. This observation can be considered as an a posteriori argument to justify neglecting the second and third terms of Eq. (3.14). The sum of these terms isn't necessarily small, and in fact for finite $p_{\max }$ it may be sizeable. The theoretical argument behind neglecting them could be that in the $p_{\max } \rightarrow \infty$ limit they vanish. However, the limit is divergent, making such an argument hard to prove.

Method of generalized expansion The method of threshold expansion, Eq. (3.12), can be straightforwardly generalized by expanding about a generic $z=z_{0}$,

$$
\begin{aligned}
C^{(k)}\left(z, \rho_{t}\right) \simeq & z^{a} \sum_{p=0}^{p_{\max }} \rho_{t}^{p} \sum_{i=0}^{i_{\max }}\left(z_{0}-z\right)^{i} \tilde{c}_{p, i}^{(k)}\left(a, z_{0}\right) \\
& +d(z) \sum_{n=0}^{k-1} A_{n}^{(k)}\left(\rho_{t}\right) \frac{\log ^{n} z}{z}
\end{aligned}
$$

where this time the damping function must be of $\mathcal{O}\left(\left(z_{0}-z\right)^{i_{\max }+1}\right)$, in order to avoid spoiling the accuracy of the expansion. Such a function can be

$d(z)=\left(1-\frac{z}{z_{0}}\right)^{i_{\max }+1} \theta\left(z_{0}-z\right)$,

where the functional form is such that in $z \rightarrow 0$ the damping is ineffective, and the theta function ensures that the small$z$ contribution remains zero for values of $z>z_{0}$ where it is forced to vanish. Equations (3.17) and (3.18) reproduce 
exactly Eqs. (3.12) and (3.13) for $z_{0}=1 .{ }^{12}$ For $z_{0}<1$, the expansion about $z_{0}$ cannot be valid in a region close to $z=1$, essentially because of the presence of logarithmic terms diverging in $z=1$ which force the convergence radius to be strictly less than $1-z_{0}$. However, this limitation can be simply overcome by patching this result with a purely threshold-expanded one at some $z=z_{1}$, with $z_{0} \leq z_{1}<1$, to be used for all $z>z_{1}$.

The advantage of this approach is that the EFT result is used in an extended region of $z$, while the contribution from the exact small- $z$ terms, which are only known at LL, is relegated to a region of smaller $z$. The limitation of this approach is that $z_{0}$ cannot be too small. Indeed, the EFT approach breaks down for $z \lesssim \rho_{t} / 4$, so $z_{0}$ must be sufficiently larger than this value. For the physical Higgs and top masses, $\rho_{t} / 4 \simeq 0.13$. An interesting value to consider is $z_{0}=1 / 2$, for two reasons. The first is that the EFT expansion parameter, $\rho_{t} /(4 z)$, equals 0.26 in $z=z_{0}=1 / 2$, which is just twice as large as its value at threshold $z=1$, and thus hopefully still sufficiently small for the EFT to be reliable. The second, more practical, is that the expansions coefficients of the leading EFT contribution $(p=0)$ have been computed for $z_{0}=1 / 2$ in the recent work of Ref. [8] up to $\mathrm{N}^{3} \mathrm{LO}$, making the implementation of this method rather straightforward.

Conclusion The considerations above bring us to the conclusion that the method of threshold expansion, Eq. (3.12), using the damping function Eq. (3.13) and $a=0$ provides the best way of implementing the correct small- $z$ logarithms in a EFT result, such as the NNLO and $\mathrm{N}^{3} \mathrm{LO}$ ones. We have implemented this method in the public code ggHiggs, version 4.0 onwards. The method of double subtraction, Eq. (3.16), has also been implemented in the code to test the sensitivity of the results on the method of including small$z$ contributions (this method was already used in previous versions of ggHiggs for the NNLO, with $a=-1$, as prescribed in Refs. [27,57]). The method of generalized expansion, Eq. (3.17), with $z_{0}=1 / 2$ has been implemented at $\mathrm{N}^{3} \mathrm{LO}$ to investigate the possibility of improving the description of the transition region $10^{-2} \lesssim z \lesssim 10^{-1}$, as we will discuss below. Instead, the method of subtraction, Eq. (3.7), due to its implementation difficulties and its arbitrariness, will be discarded.

The actual numerical implementation of the exact small$z$ logarithms has to face with the limitation that we know from resummation only the leading contribution, $A_{n}^{(k)}$ with $n=k-1$, while the coefficients with $n<k-1$ are

\footnotetext{
$\overline{12}$ To be precise, for $z_{0}=1$, the coefficients $\tilde{c}_{p, i}^{(k)}\left(a, z_{0}\right)$ must be replaced with $c_{p, i}^{(k)}(a, \ell)$ which depend on the $\log$ arithms $\ell=\log (1-z)$, so the limit $z_{0} \rightarrow 1$ isn't smooth.
}

unknown at NNLO and $\mathrm{N}^{3} \mathrm{LO}$. Here we can follow two possible approaches: we can either include only the known $A_{k-1}^{(k)}$, setting to zero all the other coefficients, or we can guess their values. Since the subleading logarithmic contributions are likely more important than the leading one in a region of medium-small $z$, keeping these coefficients certainly helps. However, exactly because they may be relevant, their values must be guessed wisely.

To do so, we follow the idea proposed in Ref. [28], namely we include subleading contributions as predicted by the LL resummation. To be precise, we use Eqs. (2.32), (2.35) and (2.36) to construct the $\mathcal{O}\left(\alpha_{s}^{2}\right)$ and $\mathcal{O}\left(\alpha_{s}^{3}\right)$ contributions to the coefficient functions in the various partonic channels in terms of the coefficients $\tilde{\mathcal{C}}_{i j}$. The anomalous dimensions $\gamma_{0,1,2}$ appearing in those equations are taken to be the expansion terms of the exact "plus" eigenvalue of the singlet anomalous dimension matrix, rather than the ones predicted by the resummation, which is appropriate for a fixed-order prediction. Finally, these expressions are expanded about $N=0$ to identify the resulting $A_{n}^{(k)}$ coefficients. This procedure is certainly not fully correct. However, in a NLL (or higher) resummation, these contributions will be part of the full prediction, which will also contain some additional correction due e.g. to the impact factor at the next perturbative order. The hope is that these corrections be less important that the contributions that we include, such that the prediction is at least reasonable.

It is clear, however, that such an implementation is not fully satisfactory, especially at $\mathrm{N}^{3} \mathrm{LO}$, where only one out of three parameters is known, i.e. $A_{2}^{(3)}$ is exact while $A_{1}^{(3)}$ and $A_{0}^{(3)}$ are only guessed. Therefore, it is important to assess, at least qualitatively, the effect of not knowing all the small$x$ contributions. To do so, we can consider variations of the unknown parameters. There are various ways in which these could be done, none of them being particularly justified. Thus, we consider only a very simple variation, which is obtained by setting to zero the coefficient of $1 / z, A_{0}^{(k)}$. At NNLO, this is the only unknown coefficient, while at $\mathrm{N}^{3} \mathrm{LO}$ it is the one that governs the contribution which has the largest impact at medium $z$, and it is thus sufficient to infer an uncertainty for those values of $z$ relevant for LHC or FCC. Incidentally, as we shall see, the resulting uncertainty covers the difference between the two implementations Eqs. (3.12) and (3.16), which is located in the medium/small- $z$ region, as well as the effect of changing $a$ from 0 to 1 or -1 . Thus, we can take the uncertainty band obtained setting $A_{0}^{(k)}=0$ as a good representative of all the small- $z$ uncertainties at fixed order.

In Fig. 2 we show the partonic coefficient functions for the gluon-gluon, gluon-quark and quark-antiquark partonic channels for factorization and renormalization scales both equal to half the Higgs mass $\left(m_{H}=125 \mathrm{GeV}\right)$, which is 

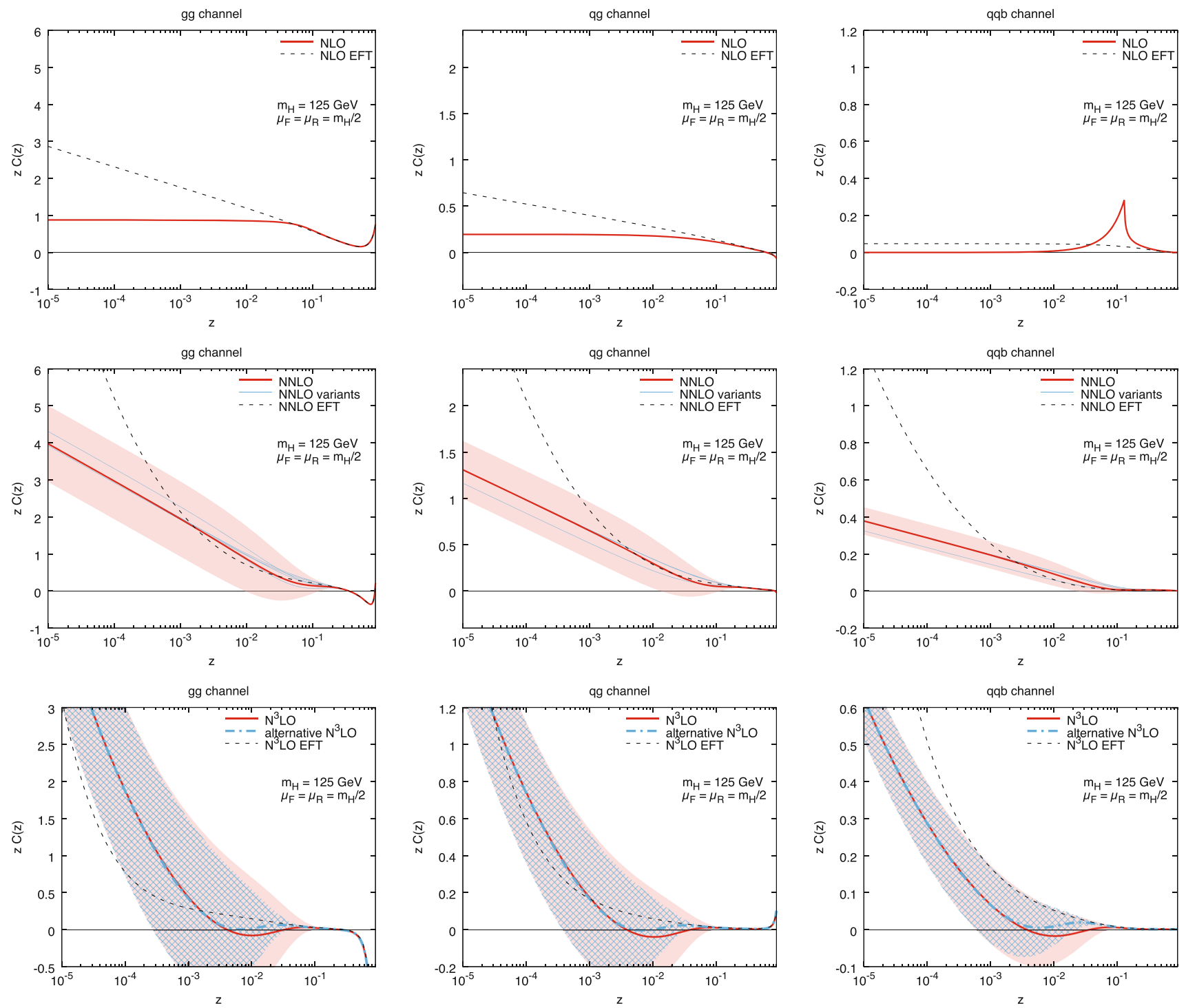

Fig. 2 Partonic coefficient functions for $g g H$ production at fixed order (upper plots: NLO; mid plots: NNLO; lower plots: $\mathrm{N}^{3} \mathrm{LO}$ ) in solid red and its EFT approximation in dashed black. In each row the three plots correspond to the $g g, q g$ and $q \bar{q}$ partonic channels. From NNLO onwards there are contributions also from other quark-quark partonic channels, which however share the same small- $z$ behaviour and are thus not shown. Fixed-order results have an uncertainty band obtained setting
$A_{0}^{(k)}=0$ and symmetrizing the variation. At NNLO some variants of the construction based on both Eqs. (3.12) and (3.16) with $a=-1,0,1$ are shown in solid light blue. At $\mathrm{N}^{3} \mathrm{LO}$ the alternative implementation Eq. (3.17) is also shown in dot-dashed blue. The Higgs mass is $m_{H}=125 \mathrm{GeV}$, the top mass $m_{t}=173 \mathrm{GeV}$, and the scales are $\mu_{\mathrm{F}}=\mu_{\mathrm{R}}=m_{H} / 2$

tively, and symmetrizing the variation with respect to our central prediction. For each plot, the leading EFT approximation $(p=0)$ is also shown in dashed black. At NNLO, we show additional curves which correspond to the two constructions Eqs. (3.12) and (3.16) with different values of $a=-1,0,1$. At $\mathrm{N}^{3} \mathrm{LO}$, the alternative implementation Eq. (3.17) is also shown, together with its own uncertainty band, in dot-dashed blue. Note that at $\mathrm{N}^{3} \mathrm{LO}$ the small- $x$ contributions are different depending on whether the $\overline{\mathrm{MS}}$ or the $Q_{0} \overline{\mathrm{MS}}$ scheme is used. Here we decide to use the $Q_{0} \overline{\mathrm{MS}}$ scheme also at fixed 
order, to match the scheme adopted in the resummed results that we will consider in the next subsection.

Several comments are in order. First, it is apparent that the EFT approximation has the wrong small- $z$ behaviour, as it exhibits double logarithmic enhancement (at leading power) rather than the correct single logarithmic enhancement. Indeed, as discussed before, the EFT is expected to fail for $z \lesssim \rho_{t} / 4 \simeq 0.13$ : this is apparent from the plots, where the red and black curves behave differently for values of $z$ smaller than about $\rho_{t} / 4$. An exception is the $q \bar{q}$ channel at NLO, where the contribution from the produced $s$-channel gluon is resonant at the $t \bar{t}$ threshold in $z=\rho_{t} / 4$, producing the peak which is clearly not present in the EFT approximation. In this case, the agreement between the EFT and exact result is restricted to a region of larger $z$. This effect is expected to be diluted at higher orders, due to the richer dynamics; nevertheless it also suggests that it is in general dangerous to trust the EFT result in the vicinity of $z=\rho_{t} / 4$.

The last comment is relevant when analysing the alternative implementation of the $\mathrm{N}^{3} \mathrm{LO}$ result based on Eqs. (3.17), (3.18) with $z_{0}=1 / 2$ and $a=0$, blue curve (and band) in the lower plots. Indeed, as expected, this construction agrees with the EFT result down to lower values of $z \gtrsim 0.05$, thus also reducing the impact of the uncertainty from subleading logarithms in the medium- $z$ region. However, the considerations above suggest that $z \sim 0.05$ is dangerously outside the region of reliability of the EFT (which is roughly speaking $z>0.2$ ), so the gain in precision (smaller uncertainty) of this construction is compensated by a loss in accuracy (the unknown exact result may lie outside the estimated uncertainty). This suggests to discard the construction based on Eq. (3.17), and use the safer construction based on Eq. (3.12).

To study the differences of the other possible alternative constructions proposed earlier in this section, we have shown in the NNLO plots some curves corresponding to variations of the $a$ parameter in our default approach Eq. (3.12), and the variant approach Eq. (3.16), again with different values of the $a$ parameter. When $a=-1$ (which we consider the lowest acceptable value, even though we favour larger values), in both approaches the soft expansion produces terms which behave as $1 / z$, and thus differ by a constant amount to our default result in Fig. 2 at small $z$. This is exactly the form of the subleading contributions used for our estimate of the uncertainty band. For $a \geq 0$, the difference is located in a region of medium $z$, approximately between $z \sim 10^{-2}$ and $z \sim 10^{-1}$. Larger values of $a>1$ (not shown in the plots) do not give any visible difference with respect to the results with $a=1$. Albeit non negligible, these variations are nicely covered by our uncertainty band, as we anticipated.

Finally, at NNLO we observe a reduction of the uncertainty band when going from $g g$ to $q g$ and to the purely quark initiated channel. This reflects a relatively less important contribution of the small- $z$ logarithms in quark channels.
At $\mathrm{N}^{3} \mathrm{LO}$ the pattern is the same, but the uncertainty bands are bigger, as appropriate due to the fact that the fraction of known small-z terms at this order is smaller. We stress that, in general, the displayed uncertainty is likely an overestimate of the actual uncertainty, since the coefficient $A_{0}^{(k)}$ is brutally set to zero rather than varied in a reasonable range. Thus, the uncertainty band will be useful only to visualize the potential impact of subleading logarithmic contributions and to motivate further work towards their computation, rather than for computing an actual uncertainty on the cross section.

\subsection{Impact of high-energy resummation at parton level}

Having described how the exact small- $z$ behaviour is included in fixed-order computations performed within the large topmass EFT framework, we now investigate the effect of supplementing the fixed-order computation with the all-order resummation of small- $z$ logarithms. At parton level, this is implemented by adding to the fixed-order coefficient functions the resummed contributions $\Delta_{k} C_{i j}$ defined in Sect. 2.4. In this section we study the impact of resummation on partonic coefficient functions, while the effect on the physical cross section will be discussed in the next section.

In Fig. 3 we report the partonic coefficient functions in the same format as Fig. 2. Results are presented at fixed order in solid red, and with resummation (in the $Q_{0} \overline{\mathrm{MS}}$ scheme) in the two implementations: using the NLL anomalous dimension in dashed blue, and using the LL' one in dot-dot-dashed yellow (see discussion in Sect. 2.2 and in Ref. [16]). The fixedorder results are also supplemented by the band which represents a rough estimate of the potential impact of unknown subleading logarithmic contributions, as described in the previous subsection. Similarly, the resummed results are supplemented by an uncertainty band, obtained varying subleading logarithmic contributions related to running coupling effects in the resummation procedure, as described in Refs. $[4,16] .{ }^{13}$

At NLO and NNLO the two implementations of the resummation give qualitatively similar results, deviating from the fixed order for $z \lesssim 10^{-1}$ at NLO and $z \lesssim 10^{-2} \div 10^{-3}$ at NNLO. The growth of the resummed contribution is slightly stronger when the NLL anomalous dimension is used. The uncertainty band of the $\mathrm{LL}^{\prime}$ variant is slightly larger than the one of the NLL variant, and covers the latter result everywhere, making them fully compatible. At NNLO, we notice that the resummed result lies within the fixed-order uncertainty band for $z \gtrsim 10^{-4} \div 10^{-3}$, which is the region most important for phenomenology. If the bands represented faithfully the uncertainty from unknown subleading logarithms

\footnotetext{
13 Specifically, we use the sum in quadrature of two independent variations, one obtained by letting $r\left(N, \alpha_{s}\right) \rightarrow \alpha_{s} \beta_{0}$ in Eq. (2.11), and the other obtained changing the way $\gamma_{+}$resums running coupling subleading contributions.
} 

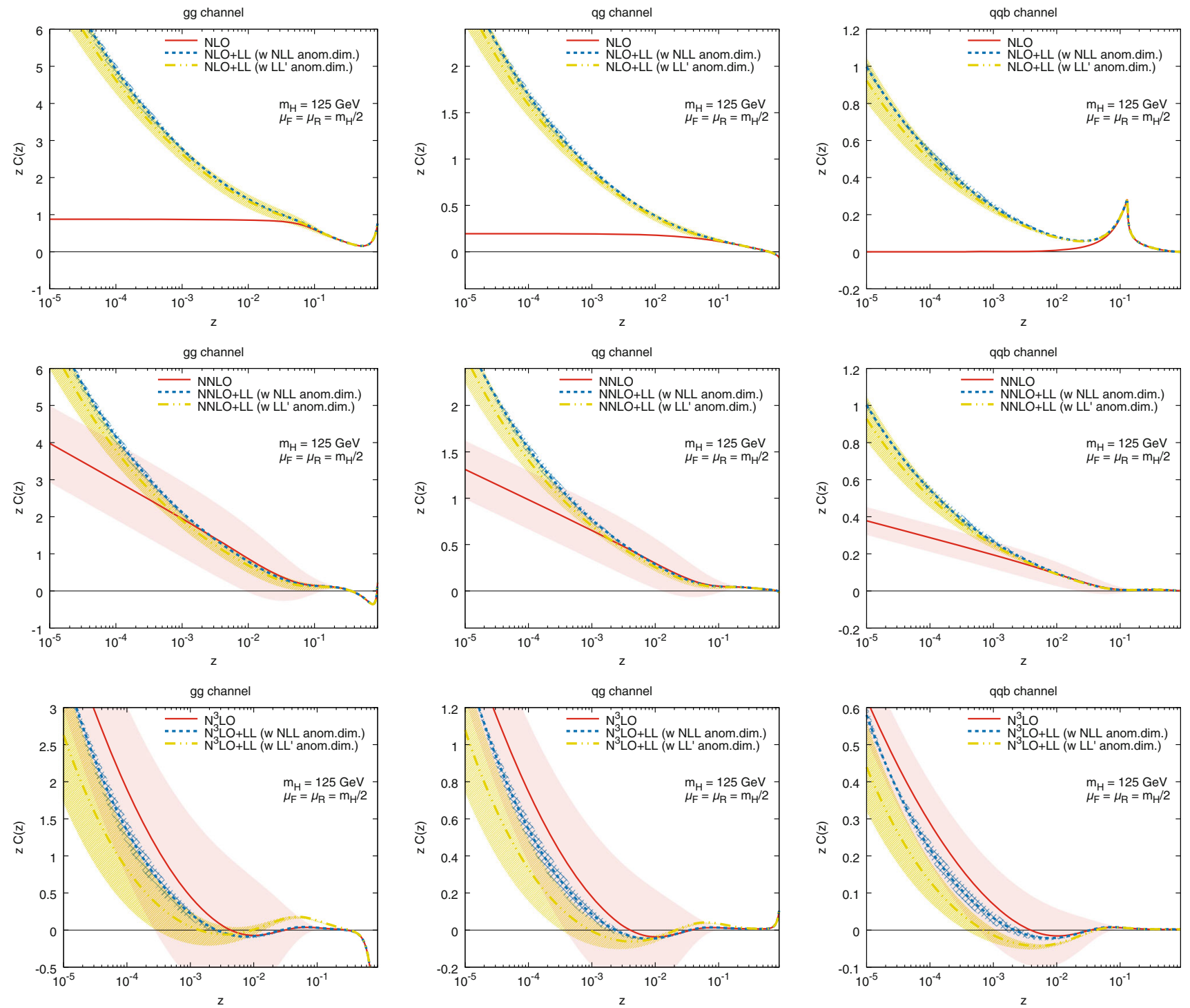

Fig. 3 Partonic coefficient functions for $g g H$ production at fixed order in solid red and fixed order plus resummation in two different implementations: dashed blue is the new implementation that uses the NLL

anomalous dimension, and dot-dot-dashed yellow is the implementation using the $\mathrm{LL}^{\prime}$ anomalous dimension. The format and details are as in Fig. 2

at fixed order, then the effect of resummation in the partonic coefficient functions would be irrelevant compared to such uncertainty.

At $\mathrm{N}^{3} \mathrm{LO}$ the general pattern is similar, with some important differences. The resummed contribution, computed using the NLL anomalous dimension, is a small correction which lies entirely within the fixed-order uncertainty band for the whole $z$ range shown. However, this time the behaviour of the resummed result with $\mathrm{LL}^{\prime}$ anomalous dimension is rather different. In general, the effect is larger than the corresponding one with NLL anomalous dimension, and no longer fully compatible with it, even though the uncertainty band is also increased. Moreover, in the $g g$ and $q g$ channels, there is a sizeable contribution of the resummation in a region of medium-large $z, 10^{-2} \lesssim z \lesssim 0.2$. This is entirely due to the $\mathcal{O}\left(\alpha_{s}^{3}\right)$ expansion of the $\mathrm{LL}^{\prime}$ anomalous dimension $\gamma_{2}^{\mathrm{LL}^{\prime}}$, Eq. (2.39), as explained in Sect. 2.5, and is indeed absent in the quark-quark channel which does not depend on it, see Eq. (2.36). This large contribution is in a region of $z$ which cannot be considered to be dominated by small- $z$ logarithms, and therefore has to be interpreted as a spurious effect. Indeed, the all-order resummed results with NLL and $\mathrm{LL}^{\prime}$ anomalous dimensions agreed in that $z$ region when matched to NLO and NNLO, so there is no physical underlying reason for which they should give such different results when matched to one order higher. 


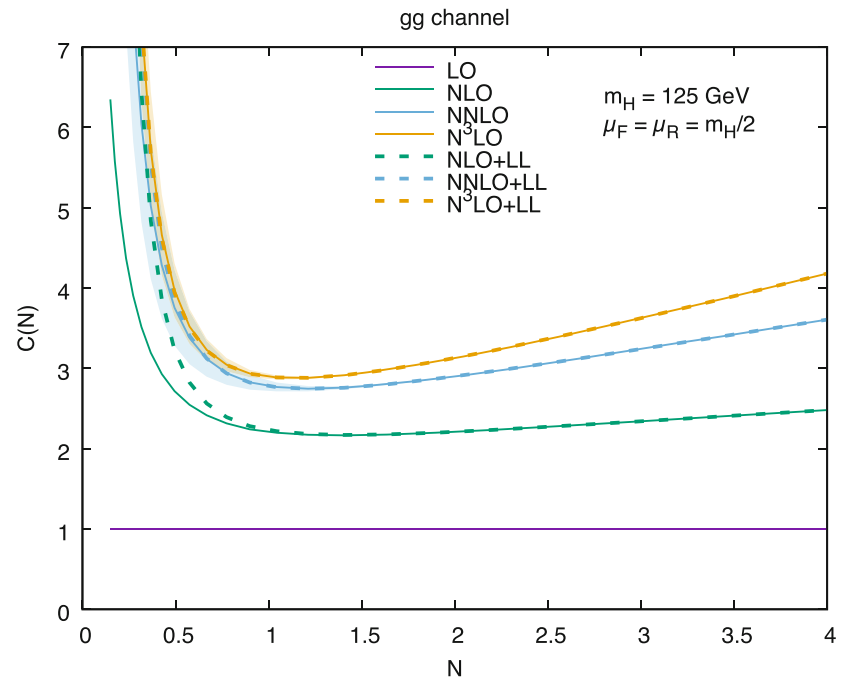

Fig. 4 Partonic $g g$-channel coefficient functions for $g g H$ production in $N$ space, at LO (solid purple), NLO (solid green), NNLO (solid blue) and $\mathrm{N}^{3} \mathrm{LO}$ (solid orange) and with resummation at NLO+LL (dashed green), NNLO+LL (dashed blue) and $\mathrm{N}^{3} \mathrm{LO}+\mathrm{LL}$ (dashed orange). The left plots shows the actual coefficient functions, while the right plot shows their ratio to the highest-order result, $\mathrm{N}^{3} \mathrm{LO}+\mathrm{LL}$. Fixed-order results are supplemented with the uncertainty band obtained setting

Our interpretation of the origin of this spurious behaviour is the fact that while the $\mathrm{LL}^{\prime}$ anomalous dimension makes perfect sense to all orders, its $\alpha_{s}$ expansion may be unstable order by order, perhaps due to its hybrid nature, and to the fact discussed in Sect. 2.5 that none of the non-vanishing contributions at $\mathcal{O}\left(\alpha_{s}^{2}\right)$ and $\mathcal{O}\left(\alpha_{s}^{3}\right)$ is exact. This is not the case for the NLL anomalous dimension, which has a well behaved $\alpha_{s}$ expansion, with the leading non-vanishing singularity correctly predicted at each order. This conclusion is in agreement with the analysis of Ref. [16], and represents another motivation for favouring the use of the NLL anomalous dimension in place of the $\mathrm{LL}^{\prime}$ one in the computation of resummed coefficient functions, in particular when these are matched to $\mathrm{N}^{3} \mathrm{LO}$ or to a higher order. Nevertheless, we must bear in mind that the resummed result based on the $\mathrm{LL}^{\prime}$ anomalous dimension differs from the NLL one by formally subleading contributions. Therefore, the difference between the two formulations probes unknown subleading logarithms. We see that this difference is similar (slightly more conservative) than the uncertainty band on the NLL-based resummed result when matched to NLO or NNLO, and could thus be used as an alternative way of estimating subleading logarithmic uncertainty. When matched to $\mathrm{N}^{3} \mathrm{LO}$, this difference is rather larger than the simple blue band, especially in the medium-large $z$ region, and using it as a subleading logarithmic uncertainty would be rather conservative. However, given that we do not really know how large these subleading logarithms may actually be, we suggest to use this difference

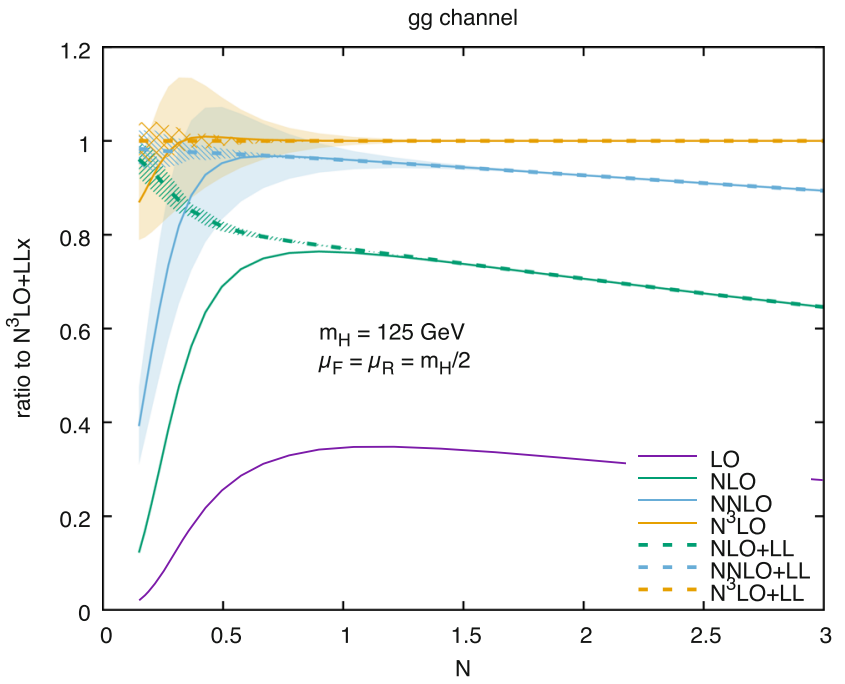

$A_{0}^{(k)}=0$ and symmetrizing the variation; the band at $\mathrm{N}^{3} \mathrm{LO}$ is just the one from the $\mathcal{O}\left(\alpha_{s}^{3}\right)$ contribution and does not contain the contribution from the previous order. Similarly, the uncertainty bands on the resummed contributions (right plot only) are estimated as the difference between the NLL and $L^{\prime} L^{\prime}$ variants of the resummation. The scales are $\mu_{\mathrm{F}}=\mu_{\mathrm{R}}=m_{H} / 2$

as a measure of such uncertainty. As we will see in the next section the resulting uncertainty at the physical cross section level is very reasonable.

Another powerful way of visualizing the effect of resummation at parton level is through the Mellin transform of the coefficient functions. In Fig. 4 we show the dominant one, $C_{g g}$, as a function of the Mellin variable $N$ for positive real $N$. This time we include the full coefficient function, and not just the regular part, since the Mellin transform of a distribution is an ordinary function. In fact, the distributional part of the coefficient function is responsible for the growth of the coefficient function at large $N$ [59]. Moreover, it is known $[38,59,60]$ that a saddle point evaluation of the Mellin inversion integral defining the full cross section (i.e. including both the coefficient functions and the PDFs) provides an excellent approximation to the exact result, thus showing that the bulk of the contribution of the coefficient function to the cross section is encoded in its value at the saddle point $N=N_{\text {saddle. }}$. From Ref. [38] we know that the saddle point for SM Higgs production varies from ${ }^{14}$ $N_{\text {saddle }} \simeq 1.1$ for LHC at $\sqrt{s}=7 \mathrm{TeV}$ to $N_{\text {saddle }} \simeq 0.9$ for LHC at $\sqrt{s}=14 \mathrm{TeV}$ and to $N_{\text {saddle }} \simeq 0.7$ for FCC at

\footnotetext{
${ }^{14}$ Note that in the mentioned references a different, more standard definition of the Mellin transform is used where the leading high-energy singularity is in $N=1$. In this work we use a different definition, common in high-energy resummation literature, where the leading singularity is in $N=0$. Thus the values of $N$ read from those references must be lowered by a unity.
} 
$\sqrt{s}=100 \mathrm{TeV}$. Thus, the region of interest for phenomenology in a vast range of hadron-hadron collider energies is all located in a small range of $N$ close to $N=1$.

In the left plot of Fig. 4 the full LO, NLO, NNLO and $\mathrm{N}^{3} \mathrm{LO}$ coefficient functions are shown, together with the resummed NLO + LL, NNLO + LL and ${ }^{3} \mathrm{LO}+$ LL counterparts. Since the plot becomes busy in the small- $N$ region, we also plot in the right panel the ratio of each curve to the highest order curve, $\mathrm{N}^{3} \mathrm{LO}+\mathrm{LL}$. We see that the resummed results depart from the fixed order for $N<1$, and they all diverge at the same $N=N_{\text {pole }}>0$, which is determined by the resummation. Thus, they all grow stronger than each fixed order, which instead are singular in $N=0$. Interestingly, the $\mathrm{N}^{3} \mathrm{LO}+\mathrm{LL}$ curve is very close to the $\mathrm{N}^{3} \mathrm{LO}$ curve even at rather small $N \gtrsim 0.2$, which is in line with the behaviour found in the $z$-space plots, and shows that the effect of small$z$ resummation on the $\mathrm{N}^{3} \mathrm{LO}$ coefficient function is expected to be negligible, since the saddle point is in a region where $\mathrm{N}^{3} \mathrm{LO}$ and $\mathrm{N}^{3} \mathrm{LO}+\mathrm{LL}$ are almost identical. In particular, the effect of subleading logarithms at fixed order, estimated by the coloured filled bands in the plots, is likely more significant than the effect of all-order resummation, both at NNLO and $\mathrm{N}^{3} \mathrm{LO}$. The fixed-order uncertainty bands also appear to be larger than the uncertainty on the resummed contributions, estimated as the difference between the NLL and LL' variants, and shown with a pattern. While we may hope, as already discussed, that these bands be over conservative, it seems important to take this observation as a strong motivation to work towards improving the knowledge of the small- $z$ behaviour of the Higgs partonic coefficient functions.

\subsection{Impact of high-energy resummation on the cross section}

We now move to the physical cross section. It is defined as the convolution of the partonic coefficient functions with the PDFs, according to Eq. (2.2) which in momentum space reads ${ }^{15}$

$$
\begin{aligned}
\sigma\left(N, Q^{2}\right)= & \sigma_{0}\left(Q^{2}\right) \sum_{i, j=g, q} \int_{\tau}^{1} \frac{d z}{z} \\
& \times C_{i j}\left(z, \alpha_{s}\left(\mu_{\mathrm{R}}^{2}\right), \frac{\mu_{\mathrm{F}}^{2}}{Q^{2}}, \frac{\mu_{\mathrm{R}}^{2}}{Q^{2}}\right) \mathscr{L}_{i j}\left(\frac{\tau}{z}, \mu_{\mathrm{F}}^{2}\right),
\end{aligned}
$$

$\mathscr{L}_{i j}\left(x, \mu_{\mathrm{F}}^{2}\right)=\int_{x}^{1} \frac{d y}{y} f_{i}\left(y, \mu_{\mathrm{F}}^{2}\right) f_{j}\left(\frac{x}{y}, \mu_{\mathrm{F}}^{2}\right)$,

\footnotetext{
15 Note that in the case of the Higgs cross section $\sigma_{0}$ is independent of $N$ in Mellin space, and thus it factors out also in the Mellin convolution in momentum space. Additionally, the sum extends over all quark flavours and not just the singlet combination.
}

with $\tau=m_{H}^{2} / s$ and $s$ che collider center-of-mass energy, and we have restored the dependence on the renormalization scale $\mu_{\mathrm{R}}$. Since high-energy resummation affects PDF evolution, and PDFs at small- $x$ are mostly determined by HERA data at low $Q^{2}$ which are thus very sensitive to resummation effects and very "far" from the Higgs scale, it is crucial to use PDFs which have been determined and evolved using resummed theory when computing physical predictions which include high-energy resummation.

Recently, such PDFs have been determined in the context of the NNPDF methodology to PDF fitting [1]. Soon after, the xFitter collaboration also performed an analogous determination [2], whose findings are in agreement with those of the NNPDF study. In both cases, PDF sets have been fitted using fixed-order theory (NLO or $\mathrm{NNLO}^{16}$ ) supplemented by highenergy resummation at NLL in the $Q_{0} \overline{\mathrm{MS}}$ scheme provided by the HELL code, version 2.0 . To be precise, resummation in DGLAP evolution is really NLL, while resummation in DIS coefficient functions is just formally NLL, since the LL contribution vanishes. In this case, we would refer to the accuracy of resummation in DIS as absolute NLL but relative LL (for this notation, see Ref. [4]). In this respect, Higgs resummation, which is relative LL, is consistent with the PDF sets of Refs. [1,2].

In fact, since the Higgs cross section is known at fixed order up to $\mathrm{N}^{3} \mathrm{LO}$, a consistent computation would require the use of PDFs obtained with $\mathrm{N}^{3} \mathrm{LO}$ theory, supplemented by resummation when computing resummed cross sections. However, this would require four-loop DGLAP splitting functions, which are not known yet, even though recently there has been some impressive progress towards their computation [35-37]. Therefore, for the time being we can only rely on NNLO (or NNLO+NLL) PDFs.

We will focus on the PDFs of Ref. [1], which are publicly available. In that work, various families of PDF sets have been obtained by using different datasets. The mainstream family is based on a global dataset, which includes on top of DIS data a large amount of "hadronic" data (mostly Drell-Yan, jet and $t \bar{t}$ production), selected in a region where resummation effects in the coefficient functions are expected to be negligible, since for these observables resummation is not yet available in HELL. Another family is then obtained by including only the DIS datasets in the fit, such that resummation is consistently included for all datapoints. Three variants of these DIS-only fits have been created by enlarging the dataset to include pseudo-data from possible future DIS experiments, namely the large hadron-electron collider ( $\mathrm{LHeC})$, the future circular electron-hadron collider (FCC-eh), and both.

For each family, four fits have been performed, with NLO, $\mathrm{NLO}+\mathrm{NLL}, \mathrm{NNLO}$ and NNLO + NLL theory (except for

\footnotetext{
16 The xFitter study [2] only considered NNLO theory, since the effects of small- $x$ resummaiton are more marked at that order.
} 
the $\mathrm{LHeC}$ and FCC families where only NNLO and NNLO + NLL is available). In all cases, the resummation makes use of the LL' anomalous dimension, which, as suggested in Ref. [16] and confirmed in this work, is not the best choice. The new version of HELL released with this work, 3.0, uses the NLL anomalous dimension rather than the $\mathrm{LL}^{\prime}$ one as default, so future fits including high-energy resummation will be performed with this new setup. So far, only a single PDF set with resummation at NNLO + NLL has been determined using the NLL anomalous dimension, which has been used in Ref. [1] to investigate the effect of subleading logarithms. However, this set is based on the DIS-only dataset, and as such it suffers from larger uncertainties and it is then not suitable for phenomenology. Nevertheless, its existence will be helpful to investigate the effect of computing consistently the Higgs cross section with our favourite choice of NLL anomalous dimension.

We have to warn the Reader that the HELL 2.0 version of the code [4] used for the aforementioned PDF fits was based on an incorrect resummation formula, which produced spurious NLL contributions to the $P_{g g}$ splitting function beyond $\mathcal{O}\left(\alpha_{s}^{3}\right)$, and affected other splitting functions and coefficient functions beyond their logarithmic accuracy. The issue has been corrected in HELL 3.0 [16]. The effect of the correction at the level of splitting functions and coefficient functions appears to be reasonably small [16], especially in the kinematic region of HERA, so we expect that the resulting PDFs are not severely affected by the issue. We stress however that the difference between the LL' and NLL formulations of the resummation matched to NLO or NNLO is significantly reduced after the correction: therefore, the non-negligible difference in the PDFs [1] obtained with these two formulations using the previous version of the code will likely be reduced significantly in future PDF fits based on HELL 3.0.

The effect of including resummation in the theory used for PDF determination is on the one hand an improvement of the quality of the description of the data, and on the other hand a rather different gluon and quark-singlet PDFs at small $x$. Such effect is much larger when resummation is added on top of the NNLO than on the NLO. The resulting gluon and quark-singlet PDFs at NNLO + NLL are harder at small- $x$ than their NNLO counterparts. The shape of the resummed PDFs is very similar in both Refs. [1,2], despite some important differences in the fitting methodology, the dataset and the treatment of the charm PDF. It is important to stress that the data constraining the PDFs at small $x$ are mostly inclusive HERA data [61] which lie at a small energy scale $Q^{2}$. The effect of small- $x$ resummation in the fit of PDFs is thus induced by the modified description of the DIS structure functions at low $x$ and $Q^{2}$, which in turn determines a different gluon and quark-singlet PDFs at low $Q^{2}$, which is then evolved to higher scales through DGLAP evolution (using resummed splitting functions). Therefore, the effect of resummation on PDFs at small $x$ at the Higgs scale is somewhat indirect (this is true also for fixed-order PDFs at small $x$ ), though not less reliable. However, it would prove very useful to include in future additional data at small $x$ and larger $Q^{2}$, e.g. from forward Drell-Yan at LHCb, to further constrain the small- $x$ PDFs at a scale closer to the Higgs scale. The resummation of such a process in HELL is work in progress.

In the rest of this section we will proceed as follows. First, we take the global PDF sets of Ref. [1] and compute predictions for the resummed Higgs cross section. Then, we will use the DIS-only PDFs to study the impact of subleading terms, both at the level of PDFs and of the coefficient functions. Additionally, in the context of the DIS-only sets we will investigate the reduction of the PDF uncertainty on the Higgs cross section that could be achieved with future DIS experiments.

Let us start with the PDFs based on the global dataset. We consider $m_{H}=125 \mathrm{GeV}$ (physical Higgs) and $m_{t}=$ $173 \mathrm{GeV}$, and compute the cross section as a function of the collider center-of-mass energy $\sqrt{s}$. We set the scales to $\mu_{\mathrm{R}}=\mu_{\mathrm{F}}=m_{H} / 2$, which is our default central choice. In Fig. 5 (left plot) we show the cross section at $\mathrm{N}^{3} \mathrm{LO}$ and $\mathrm{N}^{3} \mathrm{LO}+\mathrm{LL}$ for a range of collider energies which spans from a Tevatron-like ${ }^{17}$ energy of $\sqrt{s}=2 \mathrm{TeV}$ to a FCC-hh energy of $\sqrt{s}=100 \mathrm{TeV}$. Since the cross section changes significantly over this large range of energies, we present the results as ratios ( $K$-factors) with respect to the fixed-order $\mathrm{N}^{3} \mathrm{LO}$ prediction. For the fixed order (green) and resummed (red) predictions we use the NNLO and NNLO + NLL global PDF sets of Ref. [1], respectively. The uncertainty band shown represents the PDF uncertainty only. We see that the effect of resummation is small and compatible within the PDF uncertainty for small collider energies, up to the current LHC energy of $\sqrt{s}=13 \mathrm{TeV}$. From this value onward the net effect of the resummation is a significant increase of the cross section with respect to the fixed-order prediction, reaching up to $+10 \%$ for FCC at $\sqrt{s}=100 \mathrm{TeV}$.

This huge effect may seem surprising, and thus deserves a careful investigation. First of all, we note that basically the whole effect comes from the use of resummed PDFs, while the effect of the resummation in the coefficient function is almost negligible. Indeed, in the same plot there is an additional curve (dashed blue) obtained by computing the fixedorder $\mathrm{N}^{3} \mathrm{LO}$ cross section with the resummed PDFs: this curve, which differs from the red one only by the resummed contributions to the coefficient function, is basically identical to it, except for a tiny deviation visible only at large collider

\footnotetext{
17 We are assuming that the collider is a proton-proton collider, so this prediction is not really a Tevatron prediction. However, the difference between proton and antiproton PDFs is limited to non-singlet PDFs, which give a negligible contribution to the Higgs cross section.
} 

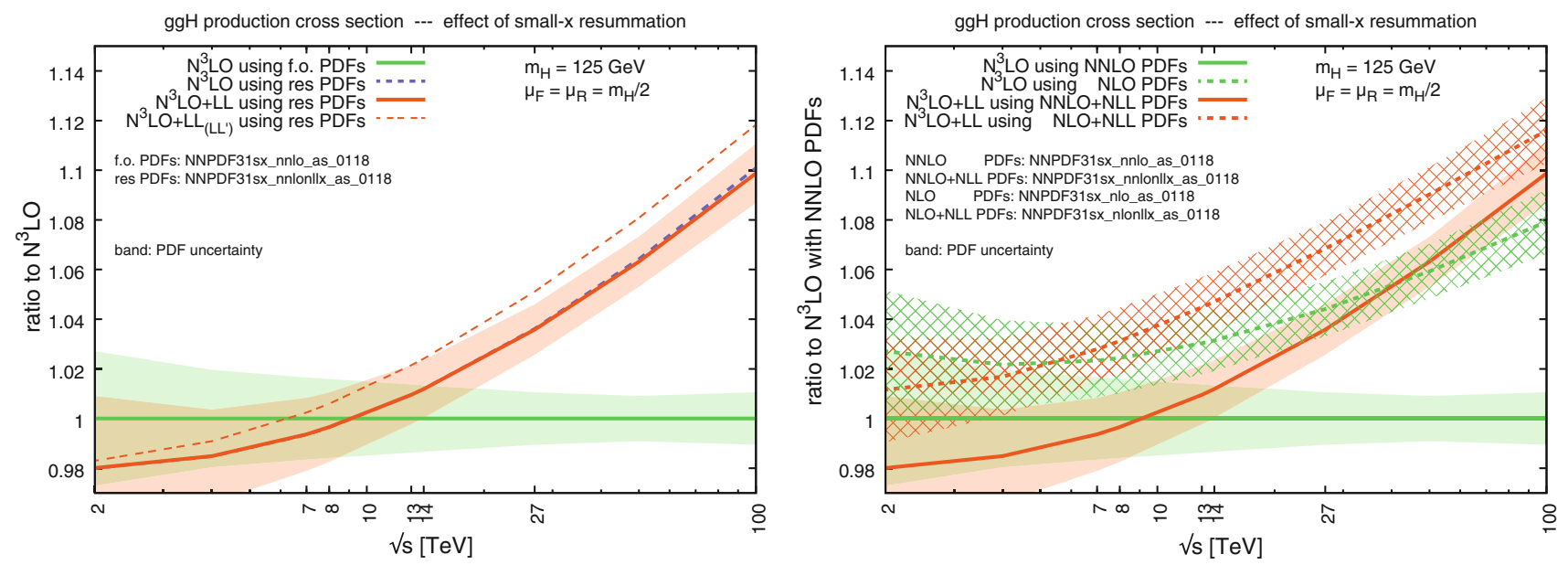

Fig. 5 Ratio of the $\mathrm{N}^{3} \mathrm{LO}$ Higgs cross section with and without resummation to the $\mathrm{N}^{3} \mathrm{LO}$ fixed-order cross section, as a function of the collider center-of-mass energy. The PDFs used are from the global dataset of Ref. [1]

energies grater than $\sqrt{s} \sim 30 \mathrm{TeV}$. These observations naturally raise the following questions. Why is the effect of high-energy resummation in the PDFs and in the partonic coefficient functions so unbalanced? Specifically, why is the effect of resummation in the PDFs so large? And why is the effect of resummation in the partonic coefficient functions so small? We now answer these three questions in turn.

The unbalance between the effect of resummation in PDFs and partonic coefficient functions is a characteristic feature of the observable under consideration being an inclusive cross section, and is due to the form of the convolution defining the cross section, Eq. (3.19). In particular, given that in the convolution when the coefficient functions are computed in $z$ the PDF luminosities are computed in $x=\tau / z$, in the integration small $z$ coefficient functions multiply large- $x$ PDFs and vice versa. To illustrate why this generates an unbalance, we show in Fig. 6 the luminosities $x \mathscr{L}_{i j}\left(x, \mu_{\mathrm{F}}^{2}\right)$ for $x=\tau / z$, as a function of the integration variable $z$, for $i j=g g$ (left plot), $i j=q g$ (middle plot) and $i j=q \bar{q}$ (right plot). These functions are the weights to the coefficient functions in the integral Eq. (3.19) defining the cross section. Since such functions depend on $\tau=m_{H}^{2} / s$, we show both the case for current $\operatorname{LHC}(\sqrt{s}=13 \mathrm{TeV}$, first line $)$ and FCC-hh $(\sqrt{s}=100 \mathrm{TeV}$, second line). It is clear that when the integration variable $z$ is small, and thus the small- $z$ logarithms are enhanced in the coefficient functions, the parton luminosities (and thus the PDFs) are computed at large values of their argument $x=\tau / z$ (reported in the upper axis), where the PDFs vanish, giving a suppressed contribution to the integral. Therefore, the region where small- $z$ resummation has an effect in the coefficient functions (roughly $z \lesssim 10^{-2}$, from Fig. 3), gives a tiny contribution to the convolution integral, i.e. to the cross section. On the contrary, the large- $z$ (threshold) region (roughly speaking, the region $z \gtrsim 0.1$ ) is enhanced in the integrand by the larger value of the luminosities and dominates the integral. ${ }^{18}$ In this region, the resummed coefficient functions reduce to their fixed-order limit (Fig. 3) and are thus insensitive to small- $z$ resummation, but the PDFs are computed at smaller values of their argument, and are thus potentially sensitive to small- $x$ logarithmic enhancement. Since this region is enhanced by the larger values of the luminosities, the effect of small- $x$ resummation in PDFs, if present, is enhanced with respect to the effect of small$z$ resummation in coefficient functions. Indeed, in the plots the luminosities are computed using both the NNLO (dashed blue) and the NNLO + NLL (solid red) sets of PDFs of Ref. [1], and it is apparent that in the FCC case, which probes smaller values of $x=\tau / z$, all the luminosities are very different at large $z$, giving the aforementioned $10 \%$ effect on the cross section. In the LHC case, the discrepancy between the two PDF sets is much less marked, but still sufficient to give the $1 \%$ effect observed in Fig. 5.

Regarding the second question, we argue that the origin of this huge difference between the predictions obtained with either the NNLO or the NNLO + NLL PDFs is due to the former being unreliable at small $x$, due to a perturbative instability in the splitting functions and DIS coefficient functions at NNLO, in turn due to the unresummed small- $x$ logarithms. Indeed, in Ref. [1] it was observed that the behaviour of the NNLO gluon PDF at small $x$ is rather different from that of the NLO PDF; the latter, in turn, is quite similar to both the NLO + NLL and NNLO + NLL resummed gluon PDFs. Namely, the perturbative progression of the PDFs is perturbatively stable at small $x$ when resummation is included, but unstable when resummation is not included, the instability

\footnotetext{
18 This enhancement of the large- $z$ portion of the integrand due to the PDF luminosities is a well known effect $[33,38,62,63]$, and it is the reason for which threshold (large-z) resummation is important for this process.
} 

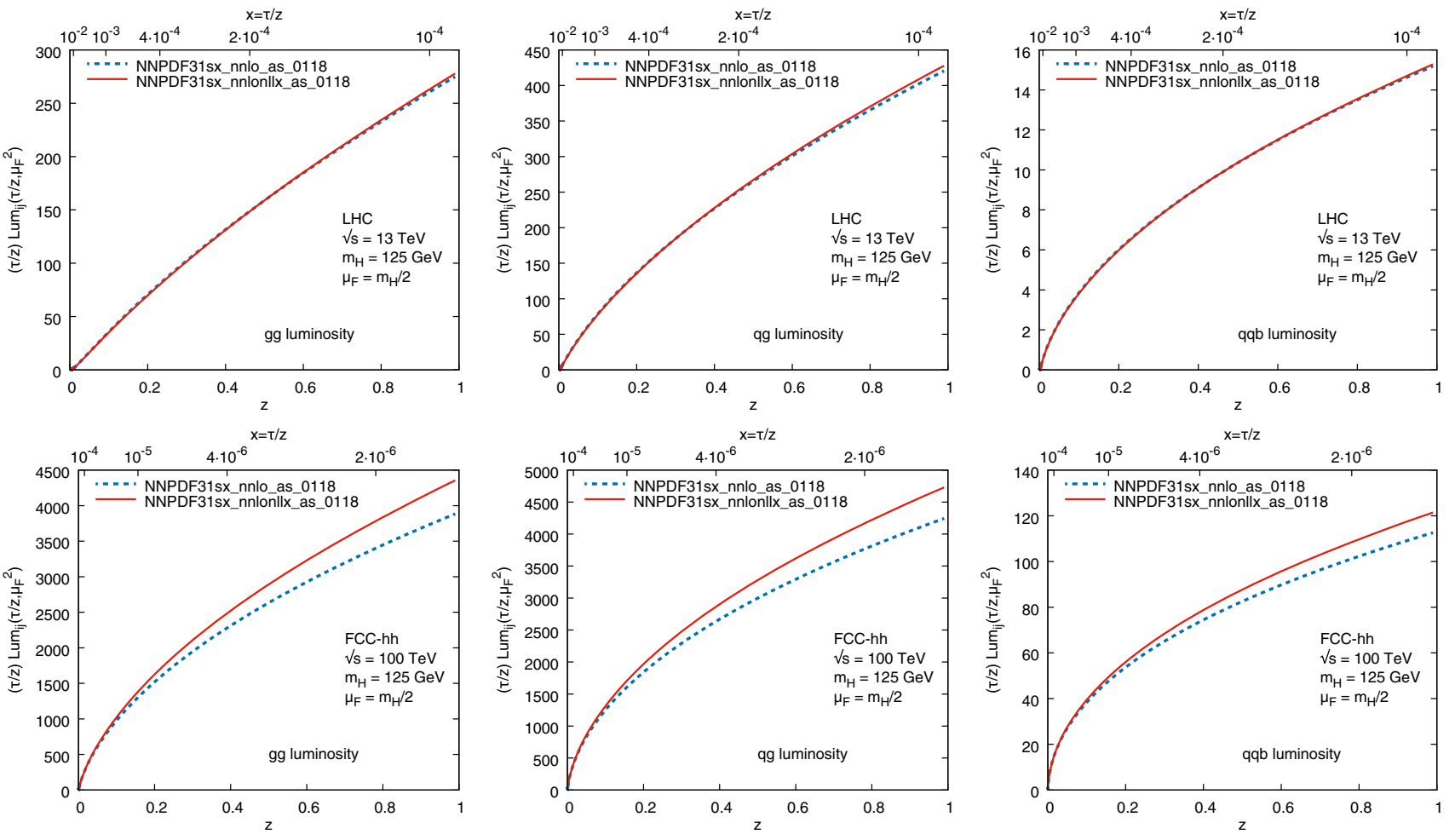

Fig. 6 The luminosities $x \mathscr{L}_{i j}\left(x, \mu_{\mathrm{F}}^{2}\right)$ for $x=\tau / z$ as a function of $z$, for $i j=g g$ (left plot), $i j=q g$ (middle plot) and $i j=q \bar{q}$ (right plot), for Higgs production at LHC at $\sqrt{s}=13 \mathrm{TeV}$ (top row) and FCC-hh at $\sqrt{s}=100 \mathrm{TeV}$ (bottom row). Values of $x=\tau / z$ are shown in the upper horizontal axis. The factorization scale corresponds to the central choice $\mu_{\mathrm{F}}=m_{H} / 2$. The PDFs used are from the global dataset of Ref. [1] starting to appear at NNLO. To understand how much of this PDF behaviour is reflected on the Higgs cross section, we show in Fig. 5 (right plot) the fixed-order and resummed cross sections using NLO PDFs (dashed green), NNLO PDFs (solid green), NLO + NLL PDFs (dashed red) and NNLO + NLL PDFs (solid red). We observe indeed that at high collider energies (which probe smaller $x$ and are thus more sensitive to small- $x$ logarithms and their resummation) all curves except the one with NNLO PDFs are grouped together, indicating that the small- $x$ instability of the NNLO is really the culprit of the huge difference between fixed-order and resummed results at high collider energies. Indeed, the resummed result with NNLO + NLL PDFs is a reasonably small correction to the results obtained with either NLO or NLO + NLL PDFs at high energies. We conclude that the effect of small- $x$ resummation on the Higgs cross section is per se not surprisingly large; however, using NNLO PDFs gives rise to unreliable results at high energies, due to the instability at small- $x$, which is not even covered by the PDF uncertainty. This effect is expected to be even more marked with $\mathrm{N}^{3} \mathrm{LO}$ PDFs, since $\mathrm{N}^{3} \mathrm{LO}$ splitting functions suffer from stronger instabilities, as demonstrated in Ref. [16]. Thus, contrary to the common lore, using $\mathrm{N}^{3} \mathrm{LO}$ PDFs for a $\mathrm{N}^{3} \mathrm{LO}$ cross section such as the Higgs cross section would produce results which are even less reliable than those with lower order PDFs. Therefore, at high energies precise and reliable predictions can only be based on small- $x$ resummed PDF sets.

We also observe that at small collider energies using resummed NNLO + NLL PDFs gives a reduction of the cross section, which seems to approach a constant value of about $-2 \%$. Here the PDF uncertainties are large, and with respect to them this effect is not significant. Moreover, the Higgs cross section at these energies is so small to be not phenomenologically relevant. Even so, it is interesting to explain the origin of this effect. This reduction of the cross section originates from a depletion of the gluon PDF for $10^{-2} \lesssim x \lesssim 10^{-1}$ when resummation is included, see e.g. Fig. 4.8 of Ref. [1]. While this effect is not genuinely a small- $x$ effect, its origin is indirectly due the inclusion of small- $x$ resummation through the constraint imposed in the PDF fits by the momentum sum rule: the smaller gluon at medium/large $x$ compensates the larger gluon at small $x$. It is then important to keep in mind that even though small- $x$ resummation has its largest effects at small $x$, the changes in the theoretical ingredients of PDF fits also induce (smaller) effects at medium and large $x$, which in turn may lead to visible effects on some observables not directly sensitive to the small- $x$ region. 
Moving to the third question, we now return to the observation that the resummation in the coefficient function has a tiny effect. This fact is partly due to the fact that we are adding resummation in the $Q_{0} \overline{\mathrm{MS}}$ scheme on top of the already rather precise $\mathrm{N}^{3} \mathrm{LO}$ prediction, and is in perfect agreement with the parton-level behaviour observed in Sect. 3.2, together with the observation that the Higgs cross section is threshold dominated, as clear from Fig. 6. However, the size of small- $z$ contributions to the coefficient functions may be different when treating differently subleading contributions, or in different factorization schemes. Indeed, we have noted in Sect. 3.2 that the partonic behaviour is rather different when $\mathrm{N}^{3} \mathrm{LO}$ is supplemented with the resummation computed with the $\mathrm{LL}^{\prime}$ anomalous dimension. In that case, the resummation has an effect also at larger $z$, and may then survive the luminosity suppression. While we believe that this effect is spurious, it is interesting to see how it affects the physical cross section. This is also interesting because the PDF sets of Ref. [1] have been obtained using resummation based on the LL' anomalous dimension. ${ }^{19}$ (However, the instability of the LL' anomalous dimension appears when expanded to $\mathcal{O}\left(\alpha_{s}^{3}\right)$, which is not the case for the NNLO + NLL resummation used in the PDFs, for which the use of the LL' formulation can be considered reliable.) Thus, in the left plot of Fig. 5 we also show the LL' version of the resummed prediction (dot-dashed red). In this case, the effect is rather large, even for small collider energies where we expect resummation to have no effect: this is entirely due to the sizeable contribution of the resummation at $z \sim 10^{-1}$ (see Fig. 3), and confirms the spurious nature of such effect. (Interestingly, the effect is positive, i.e. it does not compensate in any way the effect of the resummation in the PDFs, which would be expected if the process were included in the PDF fit.) However, it also points out that a different treatment of subleading contributions may give sizeably different results, so the smallness of the effect of resummation in the coefficient functions is also due to the specific choice of using the NLL anomalous dimension in the resummation.

While we have found strong motivations to discard the resummation based on the LL' anomalous dimension, we have suggested in Sect. 3.2 to use the difference of the resummed predictions obtained with NLL and LL' anomalous dimension as an uncertainty due to unknown subleading logarithmic contributions. This choice is certainly conservative if one considers the effect on the coefficient function alone. However, we shall not forget that subleading logarithmic contributions may have sizeable effects in the PDFs as well, which are probably not taken into account by the PDF uncertainty (see also discussion in Ref. [1]). Thus, this uncertainty has the role to also account for subleading logarithms

\footnotetext{
19 We recall that the PDF set was obtained with the previous version of HELL, and therefore the LL' is not really consistent with those PDFs.
}

in PDFs, e.g. to compensate for the fact that these PDFs have not been obtained using the NLL anomalous dimension.

It would be interesting to quantify how large the uncertainty from subleading logarithmic contributions in the PDFs can be. One way to do so is to use a PDF set which has been determined using resummation implemented through the NLL anomalous dimension. In such a PDF set both the DGLAP evolution and the theory used to describe the DIS data at small $x$ (all of which lie at small $Q^{2}$ ) differ by subleading contributions with respect to the implementation based on the $\mathrm{LL}^{\prime}$ anomalous dimension. Despite the fact that the difference is subleading, and that when resummation is matched to NNLO (as in the PDF fit) the difference between the LL' and NLL implementations is small, the effect on the resulting PDFs may be sizeable, mostly because the relevant DIS data lie at small $Q^{2}$ where higher order corrections are enhanced by large values of $\alpha_{S}\left(Q^{2}\right)$. As anticipated, in Ref. [1] a single NNLO + NLL fit based on the NLL anomalous dimension ${ }^{20}$ has been performed. As one can appreciate from Fig. 4.4 of Ref. [1], the qualitative behaviour of the PDFs and the significance of the effect of resummation is the same with both choices for the anomalous dimension. Nevertheless, the effect of subleading contributions gives a quantitatively different result, as one may expect from the argument above. This effect is not covered by the PDF uncertainty, and thus it is important to understand how it impacts a physical cross section. However, this variant of the fit was performed just in the context of the DIS-only dataset. Thus, to investigate the effects of subleading contributions in a consistent manner, we need to consider the DIS-only fits, which however suffer from larger uncertainties and are thus not suitable for phenomenological applications.

In Fig. 7 we show (left plot) the resummed cross section (normalized to the $\mathrm{N}^{3} \mathrm{LO}$ one computed with NNLO PDFs) with four different combinations of choices of subleading contributions: using consistently the $\mathrm{LL}^{\prime}$ anomalous dimension in both PDFs and coefficient functions (dot-dashed red), using the $\mathrm{LL}^{\prime}$ anomalous dimension in the PDFs and the NLL one in the coefficient functions (solid red, our default), using consistently the NLL anomalous dimension in both PDFs and coefficient functions (solid blue), and using the NLL anomalous dimension in the PDFs and the LL' one in the coefficient functions (dot-dashed blue). We restrict our attention to the LHC-FCC energy range, and show on our default prediction (solid red) the PDF uncertainty band (darker red area) and the sum in quadrature of it with the "subleading logarithmic uncertainty" as defined above, namely by the difference between solid and dot-dashed red (lighter red area).

\footnotetext{
${ }^{20}$ However, as already mentioned, also this fit was performed prior to the correction in the resummation code, where the difference between the $L^{\prime}$ and NLL variant was larger than in the bug-fixed version HELL 3. 0 .
} 


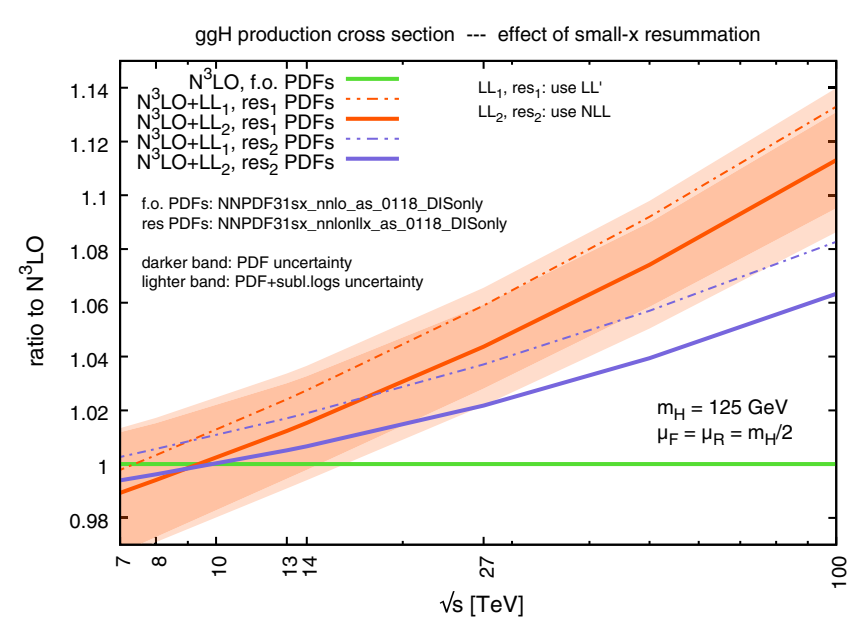

Fig. 7 Left plot: ratio of the $\mathrm{N}^{3} \mathrm{LO}+$ LL Higgs cross section to the $\mathrm{N}^{3} \mathrm{LO}$ one as a function of the collider energy, using various combinations for the implementation of resummation in the coefficient function and in the (DIS-only) PDFs. Right plot: relative PDF uncertainty as a

The solid blue curve is what we would consider the new default prediction, as it uses consistently the NLL anomalous dimension, as suggested in Ref. [16] and here. We note that such prediction is smaller than our default one, reaching "just" a $6 \%$ increase over the $\mathrm{N}^{3} \mathrm{LO}$ at $\mathrm{FCC}$, and suggesting that our current default prediction may overestimate the real effect. Nevertheless, we see that our full uncertainty band reasonably takes into account the difference between the two predictions, even though the blue curve lies outside the band for $\sqrt{s} \gtrsim 30 \mathrm{TeV}$. However, we need to keep in mind that these PDFs are based on the previous version of HELL, where the difference between LL' and NLL formulations was larger than in the new corrected version, as we commented before. We may realistically expect that with the new version of the code the PDF sets corresponding to the two variants of the resummaiton be closer to each other, such that our uncertainty band successfully covers such effect. A definitive answer can only be obtained in future, when a (possibly global) PDF fit will be performed with the new HELL 3.0 default, ideally also including the resummation of hadron-hadron collider observables, most importantly Drell-Yan cross sections, which can directly constrain small- $x$ PDFs at larger $Q^{2}$ and then reduce an unavoidable source of uncertainty coming from the large portion of DGLAP evolution from the low$Q^{2}$ HERA region (where the data which constrain the PDFs at low $x$ lie) to the ElectroWeak scale. In any case, it appears clear that subleading contributions at small $x$ are important, and should be taken into account when computing the uncertainty from missing higher orders in PDF determination.

In the context of DIS-only fits, in Ref. [1] it has been studied the impact of the inclusion of pseudo-data from possible future DIS experiments at $\mathrm{LHeC}$ and FCC-eh. It is interesting

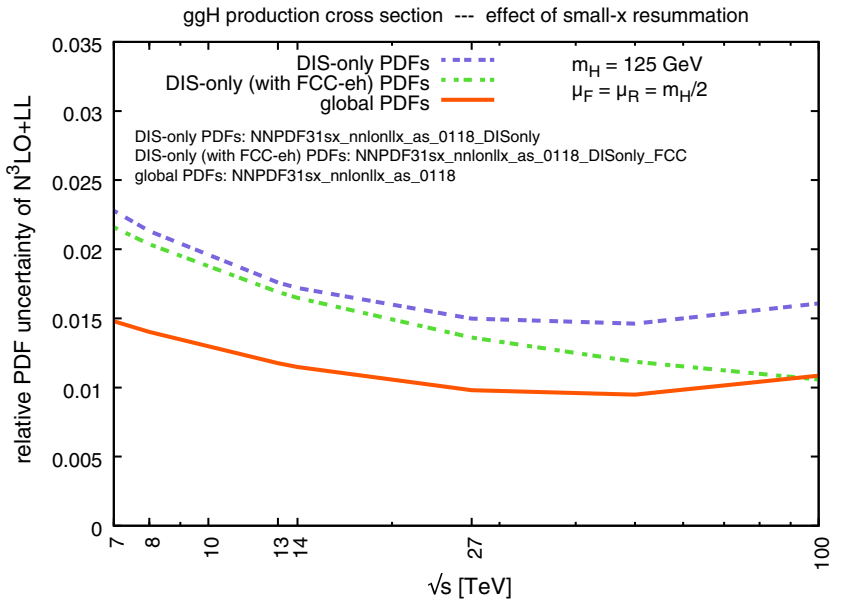

function of the collider energy for the resummed cross section obtained with the global, DIS-only and DIS-only + FCC-eh PDF sets of Ref. [1]

to use those results to study the benefits that the construction of such experiments may give in the prediction of the Higgs cross section. While this study is interesting also beyond the business of small- $x$ resummation, a striking feature of both the $\mathrm{LHeC}$ and FCC-eh datasets is to provide a significant reduction on the PDF uncertainty at small $x$, also due to the extended sensitivity to smaller values of $x$ than reached at HERA, thus also enhancing the sensitivity to small- $x$ resummation effects [1]. Most of the uncertainty reduction is provided by the FCC-eh dataset, with the $\mathrm{LHeC}$ dataset providing only an extra little improvement. Being realistic (it is unlikely that both facilities will be built) and also wanting to maximize the effect of the new data, we decide to consider the PDFs obtained with the addition of the FCCeh dataset alone. In Fig. 7 (right plot) we show the relative PDF uncertainty of the $\mathrm{N}^{3} \mathrm{LO}+\mathrm{LL}$ result for the real DISonly fit (dashed blue) and the futuristic DIS-only fit including FCC-eh pseudo-data (dot-dashed green). We see that indeed the reduction is significant and important in the high-energy region. However, it is way less dramatic than the analogous reduction visible in the gluon PDF (see Ref. [1]). This is due to the fact that we are considering an inclusive cross section, which, according to Eq. (3.19), contains contributions from all the regions of $x$ from $\tau$ to 1 . Thus, the strong uncertainty reduction on the gluon at small $x$ has only a limited benefit on the full PDF uncertainty of the cross section even at rather large collider energies. Indeed, for comparison, in the plot the uncertainty obtained with the global dataset (and thus without FCC-eh) is also shown (solid red): this uncertainty is always smaller than the DIS-only with FCC-eh one, up to the FCC-hh energy where they become comparable. Thus, for the inclusive cross section, future DIS experiments may 

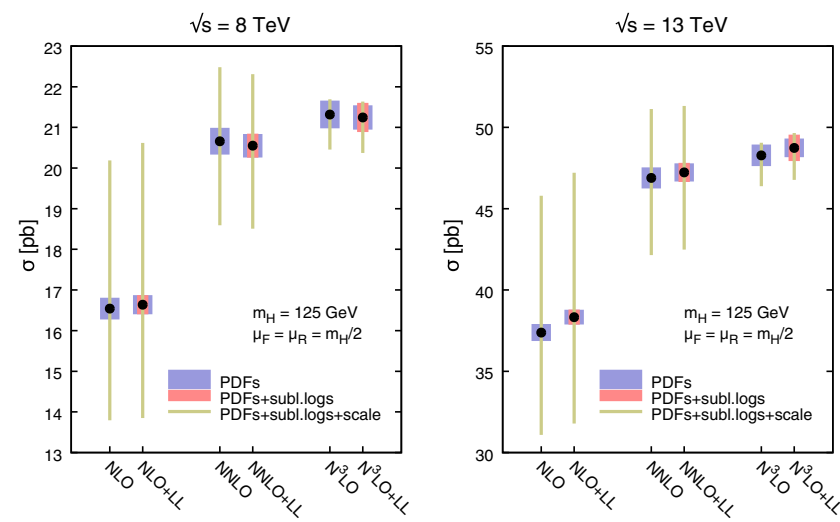

Fig. 8 Perturbative progression of the Higgs cross section for four collider energies $\sqrt{s}=\{8,13,27,100\} \mathrm{TeV}$. In each plot the NLO, $\mathrm{NLO}+\mathrm{LL}, \mathrm{NNLO}, \mathrm{NNLO}+\mathrm{LL}, \mathrm{N}^{3} \mathrm{LO}$ and $\mathrm{N}^{3} \mathrm{LO}+\mathrm{LL}$ results are shown.

lead to an increased precision at high energies, but also precise hadron collider data can, and only combining both of them one can achieve a higher precision. When considering differential observables, which are more directly sensitive to the PDFs at specific values of the momentum fraction, the uncertainty reduction provided by FCC-eh or $\mathrm{LHeC}$ may be more substantial.

So far we have presented results (with and without resummation) at $\mathrm{N}^{3} \mathrm{LO}$. To complete the discussion, we present some representative results at previous orders. In Fig. 8 we show the NLO, NNLO and $\mathrm{N}^{3} \mathrm{LO}$ cross sections, and their counterparts with resummation, for four choices of the collider energies, namely $\sqrt{s}=8 \mathrm{TeV}$ (LHC Run 1), $\sqrt{s}=13 \mathrm{TeV}$ (LHC Run 2), $\sqrt{s}=27 \mathrm{TeV}$ (HE-LHC), and $\sqrt{s}=100 \mathrm{TeV}$ (FCC-hh). We use the global NNLO PDF set for all fixed-order predictions, and the global NNLO + NLL PDFs for all resummed predictions. For each prediction we show various uncertainties. At fixed-order, the PDF uncertainty (blue) and its sum in quadrature with the (asymmetric) scale uncertainty (envelope of the standard 7-point scale variation, yellow). At resummed level, the PDF uncertainty (blue), its sum in quadrature with the uncertainty from subleading logarithms (salmon), and their sum in quadrature with the scale uncertainty (yellow). We observe that since most of the effect of the resummation is due to the PDFs, the increase in the cross section is more or less independent of the perturbative order. Therefore the perturbative progression does not improve significantly when adding resummation, ${ }^{21}$ even though a marginal improvement is anyway visible - for instance, at the FCC-hh the NLO full uncertainty band does not cover the NNLO result, while the NLO + LL band does cover the central NNLO + LL result. The scale uncertainty, being it dominated by the $\mu_{\mathrm{R}}$ dependence, is not improved

21 Threshold resummation, instead, has exactly the effect of predicting most of the higher order contributions, and thus speeds up the perturbative convergence, see Refs. [17,46].
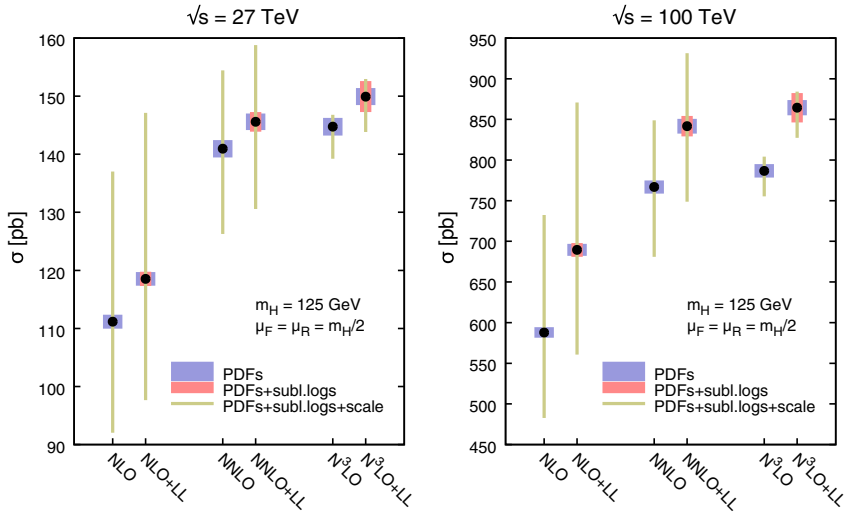

The results are supplemented by uncertainty bands from PDF, subleading logarithms and scale uncertainties

either, again because most of the resummation effect is given by the PDFs, which only depend on $\mu_{\mathrm{F}}$. It is interesting to note that the uncertainty from subleading logarithmic contributions is negligible at NLO + LL, small at NNLO + LL and quite large (comparable with scale uncertainty for HE-LHC and FCC-hh) at $\mathrm{N}^{3} \mathrm{LO}+\mathrm{LL}$. Because we compute this uncertainty as the difference between using NLL and LL' anomalous dimensions in the resummation of coefficient functions, this pattern shows that these two approaches give quantitatively similar results at NLO+LL and NNLO+LL, but as we have already noted they differ significantly at $\mathrm{N}^{3} \mathrm{LO}+\mathrm{LL}$, in agreement with the parton level results presented in Sect. 3.2.

We do not report explicit numerical results, as these have been already presented in Ref. [17], where the contribution from threshold resummation is also included, which is known to stabilize the perturbative expansion of the Higgs cross section, and additional corrections due to e.g. the bottom and charm quark running in the loop are considered. Therefore, the results of Ref. [17] are more appropriate for phenomenological applications.

We conclude the section with a final observation on the importance of considering the effect of small- $x$ resummation for precision phenomenology. At the current LHC energy including resummation leads to a $1 \%$ increase of the cross section. This effect is covered by the estimate of the theory uncertainty ${ }_{-3.6 \%}^{+1.4 \%}$ from missing higher orders (in both coefficient functions and PDFs) recommended by the LHC HXSWG [7,51]. ${ }^{22}$ However, when including additional corrections at threshold [17], the overall effect of resummations becomes an increase of $2 \%$ of the cross section, which is outside the LHC HXSWG uncertainty. This shows on the one hand that such uncertainty is likely underestimated, and on the other hand that the inclusion of resummation(s) is neces-

\footnotetext{
22 This uncertainty is the linear sum (as prescribed by Refs. [7,51]) of the uncertainty from scale variations $\left({ }_{-2.4 \%}^{+0.2 \%}\right)$ and the estimate of the uncertainty from missing higher order corrections in the PDFs $( \pm 1.2 \%)$.
} 
sary to achieve the (few) percent accuracy goal. Moving to higher collider energies, the effect of small- $x$ resummation becomes more substantial. For instance, we have seen that at the FCC-hh the effect of resummation amounts to an increase of the cross section of approximately $10 \%$. This is well outside the analogous estimate of the theory uncertainty from missing higher orders ${ }_{-4.6 \%}^{+3.5 \%}$ presented in Ref. [63], mostly due to the fact that this estimate [7] of the uncertainty from missing higher orders in the PDFs is only based on the perturbative progression at lower orders, and thus it does not take into account the presence of logarithmically enhanced contributions at small $x$, which are responsible for the sizeable effect of small- $x$ resummation to this cross section. Therefore, the inclusion of small- $x$ resummation is essential not only to reach a higher precision, but also to avoid underestimating the potential effects of higher order corrections. These considerations easily hold for other processes as well, and in some cases (e.g. differential observables more directly sensitive to small- $x$ PDFs) these effects may be much more relevant even at the $\mathrm{LHC}$.

\section{Conclusions}

In this work we have extended the resummation formalism for partonic coefficient functions originally developed for deep inelastic scattering [3] to the case of two hadron in the initial state, relevant for LHC. In particular, at the leading logarithmic accuracy we considered, only processes which are initiated by two gluons at LO, such as Higgs production in gluon fusion, top-pair production, jet production, etc., are non-trivial, while processes which are quark initiated like Drell-Yan resum only through a single initial state leg at this order, and are thus treated identically to the single-hadron case. We have demonstrated the equivalence of our (more general) approach with the original ABF approach of Ref. [9] under specific assumptions, and provided all the ingredients needed to match resummed results to fixed-order computations up to $\mathrm{N}^{3} \mathrm{LO}$. This formalism has been implemented in the new version of the public code HELL 3.0 .

We then studied a specific hadron-hadron collider process, namely Higgs production in gluon fusion. The partonic coefficient functions with incoming off-shell gluons needed for obtaining the resummed on-shell coefficient functions for this process have been computed a while ago [64-66]. However, it was possible to obtain consistent resummed predictions only thanks to two recent developments. On the one hand, the creation of the public code HELL which implements the formalism for resummation developed in Refs. [3,4] and extended to the hadron-hadron collider case in this work. On the other hand, the existence of PDF sets which have been obtained using small- $x$ resummation (from HELL) in their determination and evolution $[1,2]$.
Comparing the Higgs cross section at $\mathrm{N}^{3} \mathrm{LO}$ supplemented by small- $x$ resummation using resummed NNLO + NLL PDFs with the (current standard according to the LHC HXSWG) fixed-order $\mathrm{N}^{3} \mathrm{LO}$ prediction using NNLO PDFs, we have found that the cross section increases mildly $(+1 \%)$ at current LHC energy, and increases more substantially for larger collider energies, reaching $+4 \%$ at $\mathrm{HE}-\mathrm{LHC}$ $(\sqrt{s}=27 \mathrm{TeV})$ and $+10 \%$ at FCC-hh $(\sqrt{s}=100 \mathrm{TeV})$. In the $Q_{0} \overline{\mathrm{MS}}$ scheme that we adopt, almost all of this effect comes from the use of resummed PDFs, and in particular it is due to the fact that NNLO PDFs are unstable at small$x$ due to the presence in the three-loop splitting functions of large unresummed logarithms of $x$ [1]. The effect would be potentially much larger if (yet unavailable) $\mathrm{N}^{3}$ LO PDFs were used, since four-loop splitting functions are even more unstable due to larger powers of the logarithms at small- $x$ [16].

The main conclusion that we draw is that predictions based on NNLO PDFs and in future on $\mathrm{N}^{3} \mathrm{LO}$ PDFs will be unreliable for processes which are sensitive to small- $x$ PDFs, due to the bias induced by the perturbative instability of the splitting functions and coefficient functions of processes used for PDF determination, which is not accounted for in the way PDF uncertainties are estimated. While for the inclusive Higgs cross section this seems to be the case only at future colliders, for differential observables which are more directly sensitive to PDFs at a given momentum fraction this conclusion may hold also at the LHC in specific kinematic configurations (e.g., large rapidities). In these cases, a reassessment of the PDF uncertainties at small- $x$ is mandatory, for instance by comparing theoretical predictions obtained with PDF sets with and without small- $x$ resummation. The most reliable theoretical predictions should, in these cases, be based on small- $x$ resummed computation.

At the moment, the main limitation of small- $x$ resummation is its limited logarithmic accuracy. For DGLAP evolution, resummation is known at NLL, while for the coefficient functions it is known only at LL. In this work we have also studied the potential effect of subleading logarithmic contributions to the Higgs cross section, by computing different theoretical predictions which differ by subleading terms both in the coefficient functions and in the PDFs. The effect is potentially large, and while the qualitative conclusions of this study remain unchanged, achieving high precision requires the extension of the small- $x$ resummation formalism to higher logarithmic order. This ambitious goal is left to future work.

The new 3.0 version of HELL which contains all these new developments is publicly available for download at the address

http://www.ge.infn.it/ bonvini/hell 
It also uses a new default for the implementation of the resummation, as discusses in Ref. [16]. HELL 3.0 has been used in Ref. [17] to obtain double-resummed predictions at threshold (large $x$ ) and at high energy $(\operatorname{small} x$ ) for the Higgs cross section at LHC and beyond.

Acknowledgements I'm grateful to Simone Marzani for his encouragement to write this paper and for uncountable discussions, since part of this work was instrumental for Ref. [17]. I also want to thank Richard Ball, Stefano Forte, Giovanni Ridolfi, Juan Rojo and Luca Rottoli for various discussions and for their feedback on the manuscript. This work is supported by the Marie Skłodowska Curie grant HiPPiE@LHC.

Open Access This article is distributed under the terms of the Creative Commons Attribution 4.0 International License (http://creativecomm ons.org/licenses/by/4.0/), which permits unrestricted use, distribution, and reproduction in any medium, provided you give appropriate credit to the original author(s) and the source, provide a link to the Creative Commons license, and indicate if changes were made. Funded by SCOAP ${ }^{3}$.

\section{Appendix A: Off-shell coefficient function for Higgs pro- duction}

In this appendix we report some expressions which are needed for the actual computation of the resummed coefficient functions for $g g H$. In particular, we report the off-shell coefficient function, we explain how one can conveniently change variables for obtaining a reliable numerical integration, and we show how the $M$-expansion coefficient of the Mellin transform of the off-shell coefficient function (needed for the perturbative expansion of the resummed results) can be constructed. We stress that all the details given in this appendix, with the exception of the explicit expressions of the off-shell coefficients, are very general and can be used for other processes with two incoming off-shell gluons as well.

A.1: Off-shell coefficient function in suitable variables

The lowest order off-shell coefficient function for $g g H$ production with both gluons off-shell has been computed in Ref. [64]. Its form is

$\mathcal{C}\left(0, \xi_{1}, \xi_{2}, \alpha_{s}\right)=f_{1}\left(\xi_{1}, \xi_{2}\right)+\xi_{1} \xi_{2} f_{2}\left(\xi_{1}, \xi_{2}\right)+\mathcal{O}\left(\alpha_{s}\right)$,

with

$$
\begin{aligned}
f_{1}\left(\xi_{1}, \xi_{2}\right) & =\frac{\left|A_{1}\right|^{2}}{4\left|1-\frac{1}{4}\left(1-4 / \rho_{t}\right) s_{0}^{2}\left(\rho_{t}\right)\right|^{2}}, \\
f_{2}\left(\xi_{1}, \xi_{2}\right) & =\frac{2\left|A_{3}\right|^{2}}{4\left|1-\frac{1}{4}\left(1-4 / \rho_{t}\right) s_{0}^{2}\left(\rho_{t}\right)\right|^{2}}, \\
s_{0}\left(\rho_{t}\right) & = \begin{cases}\log \frac{1-\sqrt{1-4 / \rho_{t}}}{1+\sqrt{1-4 / \rho_{t}}}+i \pi & \rho_{t}>4 \\
2 i \sin ^{-1} \sqrt{\rho_{t} / 4} & \rho_{t} \geq 4,\end{cases}
\end{aligned}
$$

and we recall that $\rho_{t}=m_{H}^{2} / m_{t}^{2}$. The dimensionless form factors $A_{1}$ and $A_{3}$ have been computed in Refs. [65,66]. Before presenting their form, we observe that numerical integration of this function is problematic in the region $\xi_{1} \sim \xi_{2}$. Since the off-shell cross section is symmetric under the exchange of virtualities $\xi_{1} \leftrightarrow \xi_{2}$, we suggest the change of variables

$\xi_{1}=t(1+y), \quad \xi_{2}=t(1-y)$.

Thus, the integral over virtualities of a function $F\left(\xi_{1}, \xi_{2}\right)$ transforms as

$$
\begin{aligned}
& \int_{0}^{\infty} d \xi_{1} \int_{0}^{\infty} d \xi_{2} F\left(\xi_{1}, \xi_{2}\right) \\
& \quad=\int_{-1}^{1} d y \int_{0}^{\infty} d t 2 t F(t(1+y), t(1-y)) \\
& =2 \int_{0}^{1} d y \int_{0}^{\infty} d t 2 t F(t(1+y), t(1-y)),
\end{aligned}
$$

where in the last line we have assumed $F$ to be symmetric, so that the problematic region $\xi_{1}=\xi_{2}$ lies at the boundary of the integration domain and can be better integrated numerically. In terms of these variables, the form factors $[65,66]$ have a simpler form given by

$$
\begin{aligned}
A_{1}(t, y)= & \frac{C_{0}(t, y)}{\Delta_{3}}\left[\frac{4(1+2 t)}{\rho_{t}}-(1+2 t)^{2}\right. \\
& \left.+12 \frac{(1+2 t) t^{2}\left(1-y^{2}\right)}{\Delta_{3}}\right]+2 \frac{1+2 t}{\Delta_{3}} \\
& +\frac{2}{\Delta_{3}}\left[B_{0}(-t(1+y))+B_{0}(-t(1-y))\right. \\
& \left.-2 B_{0}(1)\right]\left[t-6 \frac{t^{2}\left(1-y^{2}\right)}{\Delta_{3}}\right] \\
& +\frac{2 t y}{\Delta_{3}}\left[B_{0}(-t(1+y))-B_{0}(-t(1-y))\right] \\
& \times\left[1+12 \frac{t^{2}\left(1-y^{2}\right)}{\Delta_{3}}\right], \\
A_{3}(t, y)= & \frac{C_{0}(t, y)}{\Delta_{3}}\left[\frac{8}{\rho_{t}}-4-4 t+6 \frac{(1+2 t)^{2}}{\Delta_{3}}\right]+\frac{4}{\Delta_{3}} \\
& +\left[B_{0}(-t(1+y))+B_{0}(-t(1-y))-2 B_{0}(1)\right] \\
& \times \frac{2}{\Delta_{3}}\left(1-3 \frac{1+2 t}{\Delta_{3}}\right) \\
& +\left[B_{0}(-t(1+y))-B_{0}(-t(1-y))\right] \\
& \times \frac{12 t y(1+2 t)}{\Delta_{3}^{2}},
\end{aligned}
$$

with $^{23}$

$$
\Delta_{3}=1+4 t+4 t^{2} y^{2}
$$

$\overline{{ }^{23} \text { We write } C_{0}}$ in the form given in Ref. [66], which turns out to be numerically much more stable. 


$$
\begin{aligned}
B_{0}(\zeta)= & -\sqrt{\frac{\zeta-4 / \rho_{t}}{\zeta}} \log \frac{\sqrt{\frac{\zeta-4 / \rho_{t}}{\zeta}}+1}{\sqrt{\frac{\zeta-4 / \rho_{t}}{\zeta}}-1} \\
C_{0}(t, y)= & \frac{1}{\sqrt{\Delta_{3}}}\left[\kappa\left(\delta_{0}, T_{0}\right)+\kappa\left(\delta_{+}, T_{+}\right)+\kappa\left(\delta_{-}, T_{-}\right)\right] \\
\kappa(\delta, T)= & \operatorname{Li}_{2}\left(\frac{\delta-1}{\delta-T}\right)+\operatorname{Li}_{2}\left(\frac{\delta-1}{\delta+T}\right) \\
& -\operatorname{Li}_{2}\left(\frac{\delta+1}{\delta-T}\right)-\operatorname{Li}_{2}\left(\frac{\delta+1}{\delta+T}\right)
\end{aligned}
$$

and

$$
\begin{aligned}
\delta_{0} & =\frac{1+2 t}{\sqrt{\Delta_{3}}} & T_{0} & =\sqrt{1-4 / \rho_{t}} \\
\delta_{ \pm} & =-\frac{1 \pm 2 t y}{\sqrt{\Delta_{3}}} & T_{ \pm} & =\sqrt{1+\frac{4 / \rho_{t}}{t(1 \pm y)}} .
\end{aligned}
$$

All these expressions have been coded in HELL 3.0. In some particular limits, where some of the functions fail to evaluate numerically (mostly due to the square root terms), Taylor expansions are used to overcome this problem.

In the actual definition of the resummed coefficient functions, Eqs. (2.19a) and (2.19b), the integration extends from the position of the Landau pole $\xi_{0}$ to infinity, and the off-shell coefficient appears with derivatives with respect to $\xi_{1}$ and $\xi_{2}$. The second fact is per se not a problem, except that these derivatives must be computed analytically both for speed reasons and to avoid proliferation of numerical errors. Therefore, it is useful to limit as much as possible the number of derivatives to be computed. To do so, we first observe that we do not need to treat identically the contributions from $f_{1}$ and $f_{2}$, Eq. (A.1). Indeed, in our numerical implementation we use the expression in which the derivatives act on the coefficient function for the $f_{1}$ contribution, while we use the one with derivatives on the evolution functions for the $f_{2}$ contribution. Making the notation very schematic and omitting all arguments except the virtualities, we write Eq. (2.19a) as

$$
\begin{aligned}
C_{g g}= & \int d \xi_{1} \int d \xi_{2}\left[U\left(\xi_{1}\right) U\left(\xi_{2}\right) \frac{\partial^{2} f_{1}\left(\xi_{1}, \xi_{2}\right)}{\partial \xi_{1} \partial \xi_{2}}\right. \\
& \left.+U^{\prime}\left(\xi_{1}\right) U^{\prime}\left(\xi_{2}\right) \xi_{1} \xi_{2} f_{2}\left(\xi_{1}, \xi_{2}\right)\right]
\end{aligned}
$$

where $U$ is a shorthand for $U_{\mathrm{ABF}}^{\mathrm{ht}}$, and $U^{\prime}(\xi)$ is its the derivative with respect to $\xi$. The term proportional to $f_{2}$ is then treated as described above, namely by changing variables according to Eq. (A.5) and using the symmetry to integrate only for positive $y$ 's. The contribution to $C_{g g}$ from $f_{1}$, which we call $C_{1}$ for simplicity, is instead manipulated as follows

$$
C_{1} \equiv \int d \xi_{1} \int d \xi_{2} U\left(\xi_{1}\right) U\left(\xi_{2}\right) \frac{\partial^{2} f_{1}\left(\xi_{1}, \xi_{2}\right)}{\partial \xi_{1} \partial \xi_{2}}
$$

$$
\begin{aligned}
= & \frac{1}{2} \int d y \int d t U_{+} U_{-}\left[t \frac{\partial^{2} f_{1}}{\partial t^{2}}-2 y \frac{\partial^{2} f_{1}}{\partial t \partial y}\right. \\
& \left.-\frac{1}{t} \frac{\partial}{\partial y}\left(\left(1-y^{2}\right) \frac{\partial f_{1}}{\partial y}\right)\right] \\
= & -\frac{1}{2} \int d y \int d t U_{+} U_{-}\left[2 y \frac{\partial^{2} f_{1}}{\partial t \partial y}\right. \\
& +\left(1+t(1+y) \frac{U_{+}^{\prime}}{U_{+}}+t(1-y) \frac{U_{-}^{\prime}}{U_{-}}\right) \frac{\partial f_{1}}{\partial t} \\
& \left.-\left(\frac{U_{+}^{\prime}}{U_{+}}-\frac{U_{-}^{\prime}}{U_{-}}\right)\left(1-y^{2}\right) \frac{\partial f_{1}}{\partial y}\right]
\end{aligned}
$$

where we have defined

$U_{ \pm} \equiv U(t(1 \pm y))$

and $U_{ \pm}^{\prime}$ are still derivatives with respect to the full argument. In the first step in Eq. (A.16) we have simply performed the change of variables; in the second step we have integrated by parts some contributions to remove double $t$ and double $y$ derivatives (all boundary terms vanish). At this point one can use the symmetry $y \rightarrow-y$ to restrict the integration to positive $y$ 's up to an overall factor of 2. Eq. (A.16) is what we use in the code, and provides a stable numerical evaluation of the integral, with the advantage of depending on a single second derivative of the off-shell coefficient function.

The auxiliary function Eq. (2.19b) is instead much simpler to treat. First, the $f_{2}$ term proportional to $\left|A_{3}\right|^{2}$ does not contribute, since it is multiplied by $\xi_{1} \xi_{2}$ and one of them is zero (say, $\xi_{2}=0$ ), so we have

$C_{\mathrm{aux}}=\int d \xi U(\xi) \frac{\partial f_{1}(\xi, 0)}{\partial \xi}$.

Second, there is a single derivative, which can be directly obtained from $\partial f_{1} / \partial t$ used above. In fact, the form factor becomes much simpler in the limit $\xi_{2}=0$, i.e. $y=1$,

$$
\begin{aligned}
A_{1}(t, 1)= & C_{0}(t, 1)\left[\frac{4 / \rho_{t}}{1+2 t}-1\right] \\
& +\frac{2}{1+2 t}+\frac{4 t}{(1+2 t)^{2}}\left[B_{0}(-2 t)-B_{0}(1)\right]
\end{aligned}
$$

with

$$
\begin{aligned}
C_{0}(t, 1)= & \frac{1}{1+2 t}\left[\operatorname{Li}_{2}\left(\frac{2}{1+T_{+}}\right)+\operatorname{Li}_{2}\left(\frac{2}{1-T_{+}}\right)\right. \\
& \left.-\mathrm{Li}_{2}\left(\frac{2}{1+T_{0}}\right)-\mathrm{Li}_{2}\left(\frac{2}{1-T_{0}}\right)\right],
\end{aligned}
$$

being now $T_{+}=\sqrt{1+2 /\left(\rho_{t} t\right)}$. This analytical expression is also useful for cross-checking numerically part of the results used above in the $C_{g g}$ case.

We can now discuss the implication of restricting the integration to $\xi_{1,2}>\xi_{0}=\exp \frac{-1}{\alpha_{s} \beta_{0}}$. Let us start with the onedimensional case, $C_{\text {aux }}$, Eq. (A.18). The integrand is peaked 
at $\xi \sim \mu_{\mathrm{F}}^{2} / Q^{2} \sim 1$, and drops at large $\xi$ as a negative power of $\xi$ (in this case, as $1 / \xi^{3}$ ). Thus, we do not loose precision if we approximate the integrand as

$$
\int_{\xi_{0}}^{\infty} d \xi F(\xi) \simeq \int_{\xi_{0}}^{\xi_{F}^{m+1} \xi_{0}^{-m}} d \xi F(\xi), \quad \xi_{F}=\frac{\mu_{\mathrm{F}}^{2}}{Q^{2}}
$$

where $m>0$ cuts off the large $\xi$ region which gives a negligible contribution to the integral, and $F(\xi)$ is a generic name for the integrand. In practice, we have noticed that $m=3$ is sufficiently small to guarantee numerical stability and at the same time sufficiently large to keep the important region of the integral and cut away only negligible corrections. We then split the integrand in two pieces, from $\xi_{0}$ to $\xi_{F}$ and from $\xi_{F}$ to $\xi_{F}^{m+1} \xi_{0}^{-m}$, and perform the change of variables $\xi=\xi_{F} \exp (-u)$ and $\xi=\xi_{F} \exp (m u)$ respectively:

$$
\begin{aligned}
\int_{\xi_{0}}^{\xi_{F}^{m+1} \xi_{0}^{-m}} d \xi F(\xi)= & \int_{0}^{\log \left(\xi_{F} / \xi_{0}\right)} d u \xi_{F} \\
& \times\left[e^{-u} F\left(\xi_{F} e^{-u}\right)+m e^{m u} F\left(\xi_{F} e^{m u}\right)\right] .
\end{aligned}
$$

Then we can change variable again according to $u=$ $v \log \left(\xi_{F} / \xi_{0}\right)=\frac{v}{\beta_{0} \alpha_{s}\left(\mu_{\mathrm{F}}^{2}\right)}$, and get finally

$$
\begin{aligned}
\int_{\xi_{0}}^{\infty} d \xi F(\xi) \simeq & \int_{0}^{1} \frac{d v}{\alpha_{s} \beta_{0}} \xi_{F}\left[e^{\frac{-v}{\alpha_{s} \beta_{0}}} F\left(\xi_{F} e^{\frac{-v}{\alpha_{s} \beta_{0}}}\right)\right. \\
& \left.+m e^{\frac{m v}{\alpha_{s} \beta_{0}}} F\left(\xi_{F} e^{\frac{m v}{\alpha_{s} \beta_{0}}}\right)\right],
\end{aligned}
$$

such that the integration region is in the unit hypercube (of dimension 1 in this case), and thus directly usable in standard numerical integration routines. This expression is what is used in HELL for the one-dimensional case.

In the two-dimensional case, we need to convert the two conditions $\xi_{1}>\xi_{0}$ and $\xi_{2}>\xi_{0}$ into a condition for the $y, t$ integration range. Assuming to integrate in $y$ first, the condition becomes

$$
-\left(1-\frac{\xi_{0}}{t}\right)<y<1-\frac{\xi_{0}}{t}, \quad t>\xi_{0} .
$$

The integral of a generic function $F(t, y)$, once the $y$ integration is symmetrized, can be treated as in the one-dimensional case, approximating the integral and performing subsequent changes of variables,

$$
\begin{aligned}
& \int_{\xi_{0}}^{\infty} d t \int_{0}^{1-\xi_{0} / t} d y F(t, y) \\
& \quad \simeq \int_{\xi_{0}}^{\xi_{F}^{m+1} \xi_{0}^{-m}} d t \int_{0}^{1-\xi_{0} / t} d y F(t, y) \\
& \quad=\int_{0}^{\log \left(\xi_{F} / \xi_{0}\right)} d u \xi_{F}\left[e^{-u} \int_{0}^{1-\frac{\xi_{0}}{\xi_{F}} e^{u}} d y F\left(\xi_{F} e^{-u}, y\right)\right. \\
& \left.\quad+m e^{m u} \int_{0}^{1-\frac{\xi_{0}}{\xi_{F}} e^{-m u}} d y F\left(\xi_{F} e^{m u}, y\right)\right]
\end{aligned}
$$

$$
\begin{aligned}
= & \int_{0}^{1} \frac{d v}{\alpha_{s} \beta_{0}} \int_{0}^{1} d w \xi_{F}\left[e^{\frac{-v}{\alpha_{s} \beta_{0}}}\left(1-e^{\frac{v-1}{\alpha_{s} \beta_{0}}}\right)\right. \\
& \times F\left(\xi_{F} e^{\frac{-v}{\alpha_{s} \beta_{0}}},\left(1-e^{\frac{v-1}{\alpha_{s} \beta_{0}}}\right) w\right) \\
& \left.+m e^{\frac{m v}{\alpha_{s} \beta_{0}}}\left(1-e^{\frac{-m v-1}{\alpha_{s} \beta_{0}}}\right) F\left(\xi_{F} e^{\frac{m v}{\alpha_{s} \beta_{0}}},\left(1-e^{\frac{-m v-1}{\alpha_{s} \beta_{0}}}\right) w\right)\right],
\end{aligned}
$$

where in the last step we first changed variable $y=(1-$ $\left.e^{u} \xi_{0} / \xi_{F}\right) w$ in the first $y$ integral and $y=\left(1-e^{-m u} \xi_{0} / \xi_{F}\right) w$ in the second $y$ integral, and then we used again $u=$ $v \log \left(\xi_{F} / \xi_{0}\right)$. As before, the final result is integrated in the unit hypercube (of dimension 2 in this case), and thus immediately usable for numerical integration. This expression is implemented in HELL for the two-dimensional case.

\section{A.2: Impact factor and its expansion coefficients}

We now move to the computation of the coefficients of the $M_{1,2}$ expansion of the Mellin transform of the off-shell coefficient function, Eq. (2.20). Such Mellin transform is equivalent to Eq. (A.15) after replacing

$U\left(\xi_{i}\right) \rightarrow\left(\frac{Q^{2}}{\mu_{\mathrm{F}}^{2}} \xi_{i}\right)^{M_{i}}, \quad i=1,2$

and letting $\xi_{0} \rightarrow 0$. We can thus start from Eq. (A.16) and, after integrating by parts in $t$ the last term, we arrive at (again omitting all non-crucial arguments) ${ }^{24}$

$$
\begin{aligned}
\tilde{\mathcal{C}}\left(M_{1}, M_{2}\right)= & -\frac{1}{2} \int_{-1}^{1} d y \\
& \times \int_{0}^{\infty} d t\left(\frac{Q^{2}}{\mu_{\mathrm{F}}^{2}} t\right)^{M_{1}+M_{2}}(1+y)^{M_{1}}(1-y)^{M_{2}} \\
& \times\left[y \frac{\partial^{2} f_{1}}{\partial t \partial y}+\left(1+M_{1}+M_{2}\right) \frac{\partial f_{1}}{\partial t}\right. \\
& \left.+\frac{M_{1}-M_{2}}{M_{1}+M_{2}} \frac{\partial^{2} f_{1}}{\partial t \partial y}-4 M_{1} M_{2} t f_{2}\right] .
\end{aligned}
$$

Our goal is now to expand this expression in powers of $M_{1}$ and $M_{2}$, to construct the coefficients $\tilde{\mathcal{C}}_{k j}$, Eq. (2.21). We observe however that there is a term in Eq. (A.27) which seems to give rise to negative powers of $M_{1,2}$, namely the one with $M_{1}+M_{2}$ in the denominator. When expanding $\left(t Q^{2} / \mu_{\mathrm{F}}^{2}\right)^{M_{1}+M_{2}}$ in powers of $M_{1}+M_{2}$ all terms except the zeroth order term will compensate the denominator. Thus, the only term which is potentially dangerous is the zeroth

\footnotetext{
24 Note that the integrand is no longer symmetric for $y \rightarrow-y$, unless $M_{1}$ and $M_{2}$ (which keep reference to the incoming gluon legs) are swapped simultaneously.
} 
order one, which reads

$$
-\frac{1}{2} \int_{-1}^{1} d y(1+y)^{M_{1}}(1-y)^{M_{2}} \frac{M_{1}-M_{2}}{M_{1}+M_{2}} \int_{0}^{\infty} d t \frac{\partial^{2} f_{1}}{\partial t \partial y} .
$$

But this term vanishes, since

$$
\int_{0}^{\infty} d t \frac{\partial^{2} f_{1}}{\partial t \partial y}=-\left.\frac{\partial f_{1}}{\partial y}\right|_{t=0}=0
$$

because $f_{1}$ in $t=0$ is independent of $y$. This proves that only non-negative powers of $M_{1,2}$ are produced in the expansion of Eq. (A.27), as it must. To compute the coefficients of such an expansion in a systematic way, we find it convenient to introduce the variables

$M_{ \pm}=\frac{M_{1} \pm M_{2}}{2}$

in terms of which Eq. (A.27) becomes

$$
\begin{aligned}
\tilde{\mathcal{C}}\left(M_{1}, M_{2}\right)= & \frac{1}{2} \int_{-1}^{1} d y \int_{0}^{\infty} d t \\
& \times\left(\frac{Q^{2}}{\mu_{\mathrm{F}}^{2}} t\right)^{2 M_{+}}\left(1-y^{2}\right)^{M_{+}}\left(\frac{1+y}{1-y}\right)^{M_{-}} \\
& \times\left[\frac{\partial}{\partial y}\left(y \frac{\partial f_{1}}{\partial t}\right)+2 M_{+} \frac{\partial f_{1}}{\partial t}+\frac{M_{-}}{M_{+}} \frac{\partial^{2} f_{1}}{\partial t \partial y}\right. \\
& \left.-4\left(M_{+}^{2}-M_{-}^{2}\right) t f_{2}\right] \\
= & -\frac{1}{2} \int_{0}^{1} d y \int_{0}^{\infty} d t\left(\frac{Q^{2}}{\mu_{\mathrm{F}}^{2}} t\right)^{2 M_{+}}\left(1-y^{2}\right)^{M_{+}} \\
& \times\left\{\left[\left(\frac{1+y}{1-y}\right)^{M_{-}}+\left(\frac{1-y}{1+y}\right)^{M_{-}}\right]\right. \\
& \times\left[\frac{\partial}{\partial y}\left(y \frac{\partial f_{1}}{\partial t}\right)^{+}+2 M_{+} \frac{\partial f_{1}}{\partial t}-4\left(M_{+}^{2}-M_{-}^{2}\right) t f_{2}\right] \\
& \left.+\left[\left(\frac{1+y}{1-y}\right)^{M_{-}}-\left(\frac{1-y}{1+y}\right)^{M_{-}}\right] \frac{M_{-}}{M_{+}} \frac{\partial^{2} f_{1}}{\partial t \partial y}\right\}
\end{aligned}
$$

where in the second step we have symmetrized the integration in $y$ and restricted it to positive $y$ 's. Defining

$$
\begin{aligned}
& L_{+}=\log \left[t^{2}\left(1-y^{2}\right)\right]+2 \log \frac{Q^{2}}{\mu_{\mathrm{F}}^{2}}, \\
& L_{-}=\log \left(\frac{1+y}{1-y}\right)
\end{aligned}
$$

we can expand Eq. (A.31) as

$$
\begin{aligned}
\tilde{\mathcal{C}}\left(M_{1}, M_{2}\right)= & -\int_{0}^{1} d y \int_{0}^{\infty} d t \\
& \times \sum_{a=0}^{\infty} \frac{M_{+}^{a}}{a !} L_{+}^{a} \sum_{b=0}^{\infty} \frac{M_{-}^{b}}{b !} L_{-}^{b} \frac{1+(-1)^{b}}{2}
\end{aligned}
$$

$$
\begin{aligned}
& \times\left\{\frac{\partial}{\partial y}\left(y \frac{\partial f_{1}}{\partial t}\right)+2 M_{+} \frac{\partial f_{1}}{\partial t}\right. \\
& \left.+\frac{b}{M_{+} L_{-}} \frac{\partial^{2} f_{1}}{\partial t \partial y}-4\left(M_{+}^{2}-M_{-}^{2}\right) t f_{2}\right\} \\
& =\sum_{a=0}^{\infty} \sum_{\substack{b=0 \\
b \text { even }}}^{\infty} M_{+}^{a} M_{-}^{b} c_{a, b},
\end{aligned}
$$

where the coefficients $c_{a, b}$ of the $M_{ \pm}$expansion are given by

$$
\begin{aligned}
c_{a, b}= & \frac{1}{a ! b !} \int_{0}^{1} d y \int_{0}^{\infty} d t\left\{-L_{+}^{a} L_{-}^{b} \frac{\partial}{\partial y}\left(y \frac{\partial f_{1}}{\partial t}\right)\right. \\
& -2 a L_{+}^{a-1} L_{-}^{b} \frac{\partial f_{1}}{\partial t}-\frac{b}{a+1} L_{+}^{a+1} L_{-}^{b-1} \frac{\partial^{2} f_{1}}{\partial t \partial y} \\
& +4 a(a-1) L_{+}^{a-2} L_{-}^{b} t f_{2} \\
& \left.-4 b(b-1) L_{+}^{a} L_{-}^{b-2} t f_{2}\right\} .
\end{aligned}
$$

Once these coefficients are known, they can be converted to the desired coefficients $\tilde{\mathcal{C}}_{k j}$, Eq. (2.21), through the relation

$$
\begin{aligned}
& \tilde{\mathcal{C}}_{k, j}=\frac{1}{2^{k+j}} \sum_{\substack{b=0 \\
b \text { even }}}^{k+j} c_{k+j-b, b} \\
& \times \sum_{i=\max (0, b-j)}^{\min (b, k)}(-1)^{i}\left(\begin{array}{l}
b \\
i
\end{array}\right)\left(\begin{array}{c}
k+j-b \\
k-i
\end{array}\right) .
\end{aligned}
$$

The integrals defining the coefficients Eq. (A.34) are suitable for numerical evaluation. We stress that a straightforward expansion in powers of $M_{1,2}$ of Eq. (A.15) after the replacement Eq. (A.26) suffers from a definition of the coefficients $\tilde{\mathcal{C}}_{k j}$ in terms of integrals that are not easy to perform numerically and give rise to large numerical errors. Therefore, our construction, despite being somewhat involved, has the big advantage of reducing the numerical error significantly, which was possible by exploiting the symmetry of the off-shell coefficient function. We add that the construction presented in this subsection was actually already used for computing these coefficients for Ref. [28], but it is presented in this detail here for the first time.

\section{References}

1. R.D. Ball, V. Bertone, M. Bonvini, S. Marzani, J. Rojo, L. Rottoli, Parton distributions with small-x resummation: evidence for BFKL dynamics in HERA data. Eur. Phys. J. C 78(4) 321 (2018). arXiv: 1710.05935

2. xFitter Developers' Team Collaboration, H. Abdolmaleki et al., Impact of low- $x$ resummation on QCD analysis of HERA data. arXiv:1802.00064 
3. M. Bonvini, S. Marzani, T. Peraro, Small- $x$ resummation from HELL. Eur. Phys. J. C 76(11), 597 (2016). arXiv:1607.02153

4. M. Bonvini, S. Marzani, C. Muselli, Towards parton distribution functions with small- $x$ resummation: HELL 2.0. JHEP 12, 117 (2017). arXiv: 1708.07510

5. C. Anastasiou, C. Duhr, F. Dulat, F. Herzog, B. Mistlberger, Higgs Boson gluon-fusion production in QCD at three loops. Phys. Rev. Lett. 114, 212001 (2015). arXiv: 1503.06056

6. C. Anzai, A. Hasselhuhn, M. Höschele, J. Hoff, W. Kilgore, M. Steinhauser, T. Ueda, Exact $\mathrm{N}^{3} \mathrm{LO}$ results for $q q^{\prime} \rightarrow H+X$. JHEP 07, 140 (2015). arXiv: 1506.02674

7. C. Anastasiou, C. Duhr, F. Dulat, E. Furlan, T. Gehrmann, F. Herzog, A. Lazopoulos, B. Mistlberger, High precision determination of the gluon fusion Higgs boson cross-section at the LHC. JHEP 05, 058 (2016). arXiv: 1602.00695

8. B. Mistlberger, Higgs Boson production at Hadron colliders at $\mathrm{N}^{3}$ LO in QCD. JHEP 5, 028 (2018). https://doi.org/10.1007/ JHEP05(2018)028. arXiv:1802.00833

9. R.D. Ball, Resummation of hadroproduction cross-sections at high energy. Nucl. Phys. B 796, 137-183 (2008). arXiv:0708.1277

10. R.D. Ball, S. Forte, Summation of leading logarithms at small x. Phys. Lett. B 351, 313-324 (1995). arXiv:hep-ph/9501231

11. R.D. Ball, S. Forte, Asymptotically free partons at high-energy. Phys. Lett. B 405, 317-326 (1997). arXiv:hep-ph/9703417

12. G. Altarelli, R.D. Ball, S. Forte, Factorization and resummation of small x scaling violations with running coupling. Nucl. Phys. B 621, 359-387 (2002). arXiv:hep-ph/0109178

13. G. Altarelli, R.D. Ball, S. Forte, An Anomalous dimension for small x evolution. Nucl. Phys. B 674, 459-483 (2003). arXiv:hep-ph/0306156

14. G. Altarelli, R.D. Ball, S. Forte, Perturbatively stable resummed small x evolution kernels. Nucl. Phys. B 742, 1-40 (2006). arXiv:hep-ph/0512237

15. G. Altarelli, R.D. Ball, S. Forte, Small x resummation with quarks: deep-inelastic scattering. Nucl. Phys. B 799, 199-240 (2008). arXiv:0802.0032

16. M. Bonvini, S. Marzani, Four-loop splitting functions at small $x$. JHEP 06, 145 (2018). arXiv: 1805.06460

17. M. Bonvini, S. Marzani, Double resummation for Higgs production. Phys. Rev. Lett. 120, 202003 (2018). arXiv:1802.07758

18. S. Catani, M. Ciafaloni, F. Hautmann, Gluon contributions to small$x$ heavy flavor production. Phys. Lett. B 242, 97 (1990)

19. S. Catani, M. Ciafaloni, F. Hautmann, High energy factorization and small- $x$ heavy flavour production. Nucl. Phys. B 366, 135-188 (1991)

20. J.C. Collins, R.K. Ellis, Heavy quark production in very high energy hadron collisions. Nucl. Phys. B 360, 3-30 (1991)

21. S. Catani, M. Ciafaloni, F. Hautmann, High-energy factorization in QCD and minimal subtraction scheme. Phys. Lett. B 307, 147-153 (1993)

22. S. Catani, F. Hautmann, Quark anomalous dimensions at small $\mathrm{x}$. Phys. Lett. B 315, 157-163 (1993)

23. S. Catani, F. Hautmann, High-energy factorization and small $x$ deep inelastic scattering beyond leading order. Nucl. Phys. B 427, 475524 (1994). arXiv:hep-ph/9405388

24. S. Marzani, R.D. Ball, High energy resummation of Drell-Yan processes. Nucl. Phys. B 814, 246-264 (2009). arXiv:0812.3602

25. M. Ciafaloni, D. Colferai, Dimensional regularisation and factorisation schemes in the BFKL equation at subleading level. JHEP 09, 069 (2005). arXiv:hep-ph/0507106

26. S. Marzani, R.D. Ball, P. Falgari, S. Forte, BFKL at nextto-next-to-leading order. Nucl. Phys. B 783, 143-175 (2007). arXiv:0704.2404

27. R.V. Harlander, H. Mantler, S. Marzani, K.J. Ozeren, Higgs production in gluon fusion at next-to-next-to-leading order QCD for finite top mass. Eur. Phys. J. C 66, 359-372 (2010). arXiv:0912.2104
28. R.D. Ball, M. Bonvini, S. Forte, S. Marzani, G. Ridolfi, Higgs production in gluon fusion beyond NNLO. Nucl. Phys. B 874, 746-772 (2013). arXiv:1303.3590

29. S. Moch, A. Vogt, Higher-order soft corrections to lepton pair and Higgs boson production. Phys. Lett. B 631, 48-57 (2005). arXiv:hep-ph/0508265

30. V. Ahrens, T. Becher, M. Neubert, L.L. Yang, Origin of the Large perturbative corrections to Higgs production at hadron colliders. Phys. Rev. D 79, 033013 (2009). arXiv:0808.3008

31. C. Anastasiou, C. Duhr, F. Dulat, E. Furlan, T. Gehrmann et al., Higgs boson gluon-fusion production at threshold in $N^{3} L O Q C D$. Phys. Lett. B 737, 325-328 (2014). arXiv:1403.4616

32. M. Bonvini, R. D. Ball, S. Forte, S. Marzani, G. Ridolfi, Updated Higgs cross section at approximate $\mathrm{N}^{3}$ LO. J. Phys. G41, 095002 (2014). https://doi.org/10.1088/0954-3899/41/9/095002. arXiv: 1404.3204

33. D. de Florian, J. Mazzitelli, S. Moch, A. Vogt, Approximate $\mathrm{N}^{3}$ LO Higgs-boson production cross section using physicalkernel constraints. JHEP 10, 176 (2014). https://doi.org/10.1007/ JHEP10(2014)176. arXiv:1408.6277

34. C. Anastasiou, C. Duhr, F. Dulat, E. Furlan, T. Gehrmann, F. Herzog, B. Mistlberger, Higgs boson gluon-fusion production beyond threshold in $\mathrm{N}^{3} L O$ QCD. JHEP 03, 091 (2015). arXiv:1411.3584

35. J. Davies, A. Vogt, B. Ruijl, T. Ueda, J.A.M. Vermaseren, Large$n_{f}$ contributions to the four-loop splitting functions in QCD. Nucl. Phys. B 915, 335-362 (2017). arXiv:1610.07477

36. S. Moch, B. Ruijl, T. Ueda, J.A.M. Vermaseren, A. Vogt, Four-loop non-singlet splitting functions in the planar limit and beyond. JHEP 10, 041 (2017). arXiv:1707.08315

37. A. Vogt, S. Moch, B. Ruijl, T. Ueda, J.A.M. Vermaseren, Four-loop results on anomalous dimensions and splitting functions in QCD (2018). arXiv: 1801.06085

38. M. Bonvini, S. Forte, G. Ridolfi, The threshold region for Higgs production in gluon fusion. Phys. Rev. Lett. 109, 102002 (2012). arXiv: 1204.5473

39. S. Catani, D. de Florian, M. Grazzini, P. Nason, Soft gluon resummation for Higgs boson production at hadron colliders. JHEP $\mathbf{0 7}$, 028 (2003). arXiv:hep-ph/0306211

40. V. Ahrens, T. Becher, M. Neubert, L.L. Yang, Renormalizationgroup improved prediction for Higgs production at hadron colliders. Eur. Phys. J. C 62, 333-353 (2009). arXiv:0809.4283

41. D. de Florian, M. Grazzini, M. Grazzini, Higgs production at the LHC: updated cross sections at $\sqrt{s}=8 \mathrm{TeV}$. Phys. Lett. B 718, 117 (2012). arXiv:1206.4133

42. M. Bonvini, S. Marzani, Resummed Higgs cross section at $N^{3} L L$. JHEP 1409, 007 (2014). arXiv:1405.3654

43. S. Catani, L. Cieri, D. de Florian, G. Ferrera, M. Grazzini, Threshold resummation at $\mathrm{N}^{3} \mathrm{LL}$ accuracy and soft-virtual cross sections at $\mathrm{N}^{3}$ LO. Nucl. Phys. B 888, 75-91 (2014). arXiv:1405.4827

44. M. Bonvini, L. Rottoli, Three loop soft function for $\mathrm{N}^{3} \mathrm{LL}^{\prime}$ gluon fusion Higgs production in soft-collinear effective theory. Phys. Rev. D 91(5), 051301 (2015). arXiv:1412.3791

45. T. Schmidt, M. Spira, Higgs Boson production via gluon fusion: soft-gluon resummation including mass effects. Phys. Rev. D 93(1), 014022 (2016). arXiv: 1509.00195

46. M. Bonvini, S. Marzani, C. Muselli, L. Rottoli, On the Higgs cross section at $\mathrm{N}^{3} \mathrm{LO}+\mathrm{N}^{3} \mathrm{LL}$ and its uncertainty. JHEP 08, 105 (2016). arXiv: 1603.08000

47. S. Moch, J. Vermaseren, A. Vogt, Higher-order corrections in threshold resummation. Nucl. Phys. B 726, 317-335 (2005). arXiv:hep-ph/0506288

48. E. Laenen, L. Magnea, Threshold resummation for electroweak annihilation from DIS data. Phys. Lett. B 632, 270-276 (2006). arXiv:hep-ph/0508284 
49. R.H. Boels, T. Huber, G. Yang, Four-loop nonplanar cusp anomalous dimension in $\mathrm{N}=4$ supersymmetric Yang-Mills theory. Phys. Rev. Lett. 119(20), 201601 (2017). arXiv:1705.03444

50. A. Grozin, J. Henn, M. Stahlhofen, On the Casimir scaling violation in the cusp anomalous dimension at small angle. JHEP 10, 052 (2017). arXiv:1708.01221

51. LHC Higgs Cross Section Working Group Collaboration, D. de Florian et al., Handbook of LHC Higgs cross sections: 4. Deciphering the nature of the Higgs sector. https://doi.org/10.23731/ CYRM-2017-002. arXiv:1610.07922

52. M. Spira, A. Djouadi, D. Graudenz, P.M. Zerwas, Higgs boson production at the LHC. Nucl. Phys. B 453, 17-82 (1995). arXiv:hep-ph/9504378

53. R. Bonciani, G. Degrassi, A. Vicini, Scalar particle contribution to Higgs production via gluon fusion at NLO. JHEP 11, 095 (2007). arXiv:0709.4227

54. C. Anastasiou, K. Melnikov, Higgs boson production at hadron colliders in NNLO QCD. Nucl. Phys. B 646, 220-256 (2002). arXiv:hep-ph/0207004

55. R.V. Harlander, W.B. Kilgore, Next-to-next-to-leading order Higgs production at hadron colliders. Phys. Rev. Lett. 88, 201801 (2002). arXiv:hep-ph/0201206

56. V. Ravindran, J. Smith, W.L. van Neerven, NNLO corrections to the total cross-section for Higgs boson production in hadron hadron collisions. Nucl. Phys. B 665, 325-366 (2003). arXiv:hep-ph/0302135

57. R.V. Harlander, K.J. Ozeren, Finite top mass effects for hadronic Higgs production at next-to-next-to-leading order. JHEP 11, 088 (2009). arXiv:0909.3420

58. A. Pak, M. Rogal, M. Steinhauser, Finite top quark mass effects in NNLO Higgs boson production at LHC. JHEP 02, 025 (2010). arXiv:0911.4662

59. M. Bonvini, Resummation of soft and hard gluon radiation in perturbative QCD. PhD thesis, Genoa U (2012). arXiv:1212.0480
60. M. Bonvini, S. Forte, G. Ridolfi, L. Rottoli, Resummation prescriptions and ambiguities in SCET vs. direct QCD: Higgs production as a case study. JHEP 1501, 046 (2015). arXiv: 1409.0864

61. ZEUS, H1 Collaboration, H. Abramowicz et al., Combination of measurements of inclusive deep inelastic $e^{ \pm} p$ scattering cross sections and QCD analysis of HERA data. Eur. Phys. J. C 75(12), 580 (2015). arXiv: 1506.06042

62. T. Becher, M. Neubert, G. Xu, Dynamical threshold enhancement and resummation in Drell-Yan production. JHEP 07, 030 (2008). arXiv:0710.0680

63. R. Contino et al., Physics at a $100 \mathrm{TeV}$ pp collider: Higgs and EW symmetry breaking studies. CERN Yellow Report, no. 3, 255-440 (2017). arXiv: 1606.09408

64. S. Marzani, R.D. Ball, V. Del Duca, S. Forte, A. Vicini, Higgs production via gluon-gluon fusion with finite top mass beyond next-to-leading order. Nucl. Phys. B 800, 127-145 (2008). arXiv:0801.2544

65. V. Del Duca, W. Kilgore, C. Oleari, C. Schmidt, D. Zeppenfeld, Gluon fusion contributions to $\mathrm{H}+2$ jet production. Nucl. Phys. B 616, 367-399 (2001). arXiv:hep-ph/0108030

66. R.S. Pasechnik, O.V. Teryaev, A. Szczurek, Scalar Higgs boson production in a fusion of two off-shell gluons. Eur. Phys. J. C 47, 429-435 (2006). arXiv:hep-ph/0603258 